\title{
Removal energies and final state interaction in lepton nucleus scattering
}

\author{
Arie Bodek ${ }^{\mathrm{a}}$, Tejin Cai \\ Department of Physics and Astronomy, University of Rochester, Rochester, NY 14627-0171, USA
}

Received: 29 January 2018 / Accepted: 4 March 2019 / Published online: 1 April 2019

(C) The Author(s) 2019

\begin{abstract}
We investigate the binding energy parameters that should be used in modeling electron and neutrino scattering from nucleons bound in a nucleus within the framework of the impulse approximation. We discuss the relation between binding energy, missing energy, removal energy $(\epsilon)$, spectral functions and shell model energy levels and extract updated removal energy parameters from ee' $\mathrm{p}$ spectral function data. We address the difference in parameters for scattering from bound protons and neutrons. We also use inclusive e-A data to extract an empirical parameter $U_{F S I}\left(\left(\boldsymbol{q}_{3}+\boldsymbol{k}\right)^{2}\right)$ to account for the interaction of final state nucleons (FSI) with the optical potential of the nucleus. Similarly we use $V_{\text {eff }}$ to account for the Coulomb potential of the nucleus. With three parameters $\epsilon, U_{F S I}\left(\left(\boldsymbol{q}_{3}+\boldsymbol{k}\right)^{2}\right)$ and $V_{\text {eff }}$ we can describe the energy of final state electrons for all available electron QE scattering data. The use of the updated parameters in neutrino Monte Carlo generators reduces the systematic uncertainty in the combined removal energy (with FSI corrections) from \pm 20 to $\pm 5 \mathrm{MeV}$.
\end{abstract}

\section{Introduction}

The modeling of neutrino cross sections on nuclear targets is of great interest to neutrino oscillations experiments. Neutrino Monte Carlo (MC) generators include GENIE [1,2], NEUGEN [3], NEUT [4], NUWRO [5,6] and GiBUU [7].

Although more sophisticated models are available [8-16], calculations using a one-dimensional momentum distribution and an average removal energy parameter are still widely used. One example is the simple relativistic Fermi gas (RFG) model.

The RFG model does not describe the tails in the energy distribution of the final state lepton very well [17-20]. Improvements to the RFG model such as a better momentum distribution are usually made within the existing Monte Carlo

\footnotetext{
a e-mail: bodek@pas.rochester.edu
}

(MC) frameworks. All RFG-like models with one dimensional nucleon momentum distributions require in addition removal energy parameters $\left(\epsilon^{P, N}\right)$ to account for the average removal energy of a proton or neutron from the nucleus. These parameters should be approximately the same for all one-dimensional momentum distributions.

Alternatively two dimensional spectral functions (as a function of nucleon momentum and missing energy) can be used. However, even in this case, MC generators currently used in neutrino oscillations experiments do not account for the final state interaction (FSI) of the final state lepton and nucleon in the optical and Coulomb potentials of the nucleus.

In this paper we extract empirical average removal energy parameters from spectral function measured in exclusive ee' $p$ electron scattering experiments on several nuclei. We use $V_{\text {eff }}$ (see Appendix A) to account for the Coulomb potential of the nucleus, and extract empirical nucleon final state interaction parameter $U_{F S I}\left(\left(\boldsymbol{q}_{3}+\boldsymbol{k}\right)^{2}\right)$ from all available inclusive e-A electron scattering data. With these three parameters $\epsilon$, $U_{F S I}\left(\left(\boldsymbol{q}_{3}+\boldsymbol{k}\right)^{2}\right)$ and $V_{\text {eff }}$ we can describe the energy of final state electrons for all available electron QE scattering data. These parameters can be used to improve the predictions of current neutrino MC event generators such as GENIE and NEUT for the final state muon and nucleon energies in QE events.

A large amount of computer time has been used by various experiments to generate and reconstruct simulated neutrino interactions using MC generators such as GENIE 2. We show how approximate post-facto corrections could be applied to these existing $\mathrm{MC}$ samples to improve the modeling of the reconstructed muon, final state proton, and unobserved energy in quasielastic (QE) events.

\subsection{Relevance to neutrino oscillations experiments}

In a two neutrinos oscillations framework the oscillation parameters which are extracted from long baseline experi- 
ments are the mixing angle $\vartheta$ and the square of the difference in mass between the two neutrino mass eigenstates $\Delta m^{2}$. A correct modeling of the reconstructed neutrino energy is very important in the measurement of $\Delta m^{2}$. In general, the resolution in the measurement of energy in neutrino experiments is much worse than the resolution in electron scattering experiments. However, a precise determination of $\Delta m^{2}$ is possible if the MC prediction for average value of the experimentally reconstructed neutrino energy is unbiased. At present the uncertainty in the value of the removal energy parameters is a the largest source of systematic error in the extraction of the neutrino oscillation parameter $\Delta m^{2}$ (as shown below).

The two-neutrino transition probability can be written as

$$
P_{v_{\alpha} \rightarrow v_{\beta}}(L)=\sin ^{2} 2 \vartheta \sin ^{2}\left(1.27 \frac{\left(\Delta m^{2} / \mathrm{eV}^{2}\right)(L / \mathrm{km})}{\left(E_{v} / \mathrm{GeV}\right)}\right) .
$$

Here, $\mathrm{L}$ (in $\mathrm{km}$ ) is the distance between the neutrino source and the detector and $\Delta m^{2}$ is in $\mathrm{eV}^{2}$.

The location of the first oscillation maximum in neutrino energy $\left(E_{v}^{1 s t-\min }\right)$ is when the term in brackets is equal to $\pi / 2$. An estimate of the extracted value of $\Delta m^{2}$ is given by:

$\Delta m^{2}=\frac{2 E_{v}^{1 s t-m i n}}{1.27 \pi L}$.

For example, for the $\mathrm{T} 2 \mathrm{~K}$ experiment $L=295 \mathrm{Km}$, and $E_{v}$ is peaked around $0.6 \mathrm{GeV}$. For the normal hierarchy the $\mathrm{T} 2 \mathrm{~K}$ experiment [21] reports a value of

$$
\begin{aligned}
\Delta m_{32}^{2}(\mathrm{~T} 2 \mathrm{~K}-2018) & =(2.434 \pm 0.064) \times 10^{-3} \mathrm{eV}^{2} . \\
\sin ^{2} \theta_{23}(\mathrm{~T} 2 \mathrm{~K}-2018) & =0.536_{-0.045}^{+0.031}
\end{aligned}
$$

Using Eqs. 2 and 40 we estimate that a $+20 \mathrm{MeV}$ change in the removal energy used in the $\mathrm{MC}$ results in a change in $\Delta m_{32}^{2}$ of $+0.03 \times 10^{-3} \mathrm{eV}^{2}$, which is the largest contribution to the total systematic error in $\Delta m_{32}^{2}$.

The above estimate is consistent with the estimate of the $\mathrm{T} 2 \mathrm{~K}$ collaboration. The $\mathrm{T} 2 \mathrm{~K}$ collaboration reports $[22,23]$ that "for the statistics of the 2018 data set, a shift of $20 \mathrm{MeV}$ in the binding energy parameter introduces a bias of $20 \%$ for $\sin ^{2} \theta_{23}$ and $40 \%$ for $\Delta m_{32}^{2}$ with respect to the size of the systematics errors, assuming maximal $\sin ^{2} \theta_{23}$ ".

For the case of normal hierarchy a combined analysis [25] of the world's neutrino oscillations data in 2018 finds a best fit of

$$
\begin{aligned}
\Delta m_{32}^{2}(\text { COMBINED }-2018) & =(2.50 \pm 0.03) \times 10^{-3} \mathrm{eV}^{2}, \\
\sin ^{2} \theta_{23}(\text { COMBINED }-2018) & =0.547_{-0.030}^{+0.020},
\end{aligned}
$$

which illustrates the importance of using a common definition of removal energy parameters and the importance in handling the correlations in the uncertainties between various experiments when performing a combined analysis.
For comparison, we find that a change of $+20 \mathrm{MeV} / \mathrm{c}$ in the assumed value of the Fermi momentum $k_{F}$ yields a much smaller change of $+0.005 \times 10^{-3} \mathrm{eV}^{2}$ in the extracted value of $\Delta m_{32}^{2}$.

\subsection{Neutrino near detectors}

In general, neutrino oscillations experiments use data taken from a near detector to reduce the systematic error from uncertainties in the neutrino flux and in the modeling of neutrino interactions. However, near detector data cannot constrain the absolute energy scale of final state muons and protons, or account for the energy that goes into the undetected nuclear final state. These issues are addressed in this paper.

\subsection{Simulation of QE events and reconstruction of neutrino energy}

In order to simulate the reconstruction of neutrino QE events within the framework of the impulse approximation the experimental empirical parameters that are used should describe:

1. The momentum of the final state muon including the effect of Coulomb corrections [24].

2. The mass, excitation energy, and recoil energy of the spectator nuclear state.

3. The effect of the interaction of the final state nucleon (FSI) with the optical and Coulomb potential of the spectator nucleus.

\subsection{Nucleon momentum distributions}

Figure 1 shows a few models for the nucleon momentum distributions in the ${ }_{6}^{12} \mathrm{C}$ nucleus. The solid green line (labeled Global Fermi gas) is the nucleon momentum distribution for the Fermi gas [17] model which is currently implemented in all neutrino event generators and is related to global average density of nucleons. The solid black line is the projected momentum distribution of the Benhar-Fantoni [8-11] 2D spectral function as implemented in NUWRO. The solid red line is the nucleon momentum distribution for the LocalThomas-Fermi (LTF) gas which is is related to the local density of nucleons in the nucleus and is implemented in NEUT, NUWRO and GiBUU.

A more sophisticated formalism is the $\psi^{\prime}$ superscaling model [26,27], which is only valid for QE scattering. It can be used to predict the kinematic distribution of the final state muon but does not describe the details of the hadronic final state. Therefore, it has not been implemented in neutrino MC generators. However, the predictions of the $\psi^{\prime}$ superscaling model can be approximated with an effective spectral function [28] which has been implemented in GENIE. The 


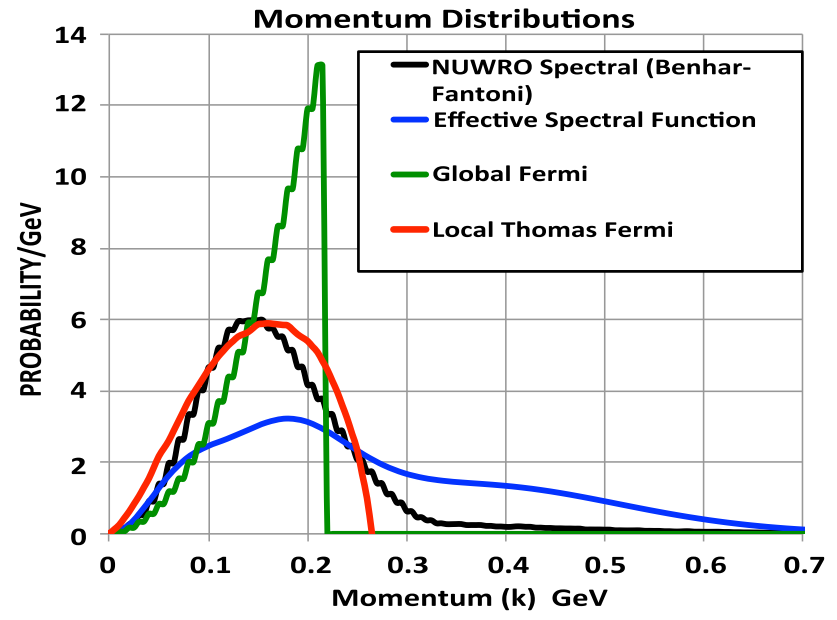

Fig. 1 One-dimensional nucleon momentum distributions in a ${ }_{6}^{12} \mathrm{C}$ nucleus. The green curve (Global Fermi) is the momentum distribution for the relativistic Fermi gas (RFG) model. The red curve is the Local-Thomas-Fermi (LTM) gas distribution. The black curve is the projected momentum distribution of the Benhar-Fantoni two dimensional spectral function. The blue line is the momentum distribution for the effective spectral function model, which approximates the $\psi^{\prime}$ superscaling prediction for the final state muon in quasielastic scattering

momentum distribution of the effective spectral function for nucleons bound in ${ }_{6}^{12} \mathrm{C}$ is shown as the blue curve in Fig. 1.

Although the nucleon momentum distributions are very different for the various models, the predictions for the normalized quasielastic neutrino cross section $\frac{1}{\sigma} \frac{d \sigma}{d v}\left(Q^{2}, v\right)$ are similar as shown in Fig. 2. These predictions as a function of $v=E_{\nu}-E_{\mu}$ are calculated for $10 \mathrm{GeV}$ neutrinos on ${ }_{6}^{12} \mathrm{C}$ at $Q^{2}=0.5 \mathrm{GeV}^{2}$. The prediction with the local Fermi gas distribution are similar to the prediction of the BenharFantoni two dimensional spectral function as implemented in NUWRO. Note that the prediction of the $\psi^{\prime}$ superscaling model are based on fits to longitudinal QE differential cross sections. Subsequently, they includes $1 \mathrm{p} 1 \mathrm{~h}$ and some $2 \mathrm{p} 2 \mathrm{~h}$ processes (discussed in Sect. 2).

The following nuclear targets are (or were) used in neutrino experiments: Carbon (scintillator) used in the NOVA and MINERVA experiments. Oxygen (water) used in T2K and in MINERVA. Argon used in the ARGONEUT and DUNE experiments. Calcium (marble) used in CHARM. Iron used in MINERUA, MINOS, CDHS, NUTEV, and CCFR. Lead used in CHORUS and MINERUA.

\section{The impulse approximation}

\section{$2.11 \mathrm{p} 1 \mathrm{~h}$ process}

Figure 3 is a descriptive diagram for $\mathrm{QE}$ electron scattering on an off-shell proton which is bound in a nucleus of mass $M_{A}$, and is moving in the mean field (MF) of all other

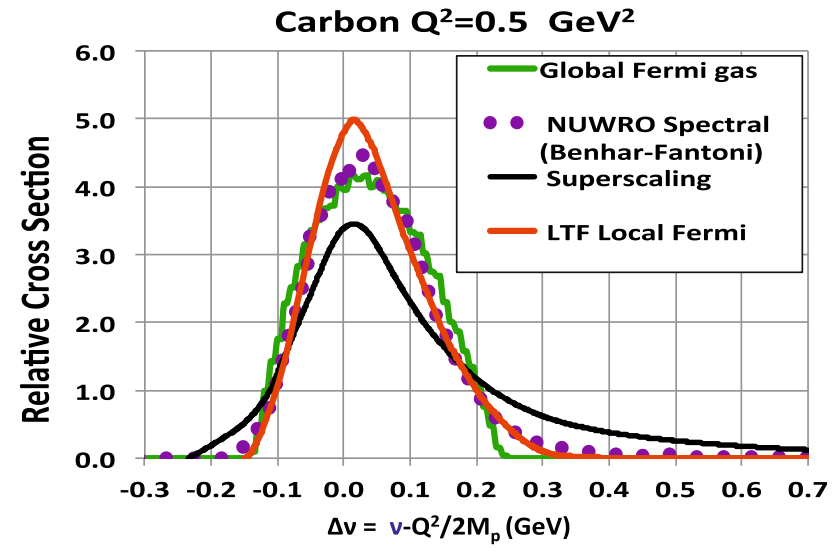

Fig. 2 Comparison of the $\psi^{\prime}$ superscaling prediction (solid black line) for the normalized quasielastic $\frac{1}{\sigma} \frac{d \sigma}{d v}\left(Q^{2}, v\right)$ at $Q^{2}=0.5 \mathrm{GeV}^{2}$ for $10 \mathrm{GeV}$ neutrinos on ${ }_{6}^{12} \mathrm{C}$ to the predictions with several momentum distribution $\left(v=E_{0}-E^{\prime}\right)$. Here the solid green curve labeled "Global Fermi" gas is the distribution for the Fermi gas model. The red line is the prediction for the local Thomas Fermi (LTF) gas, and the purple dots are the prediction using the two dimensional Benhar-Fantoni spectral function as implemented in NUWRO

nucleons in the nucleus. The on-shell recoil excited $[A-1]^{*}$ spectator nucleus has a momentum $\boldsymbol{p}_{(A-1) *}=-\boldsymbol{k}$ and a mean excitation energy $\left\langle E_{x}^{P}\right\rangle$. The off-shell energy of the interacting nucleon is $E_{i}=M_{A}-\sqrt{\left(M_{A-1} *\right)^{2}+\boldsymbol{k}^{2}}=$

\section{Electron scattering on proton}

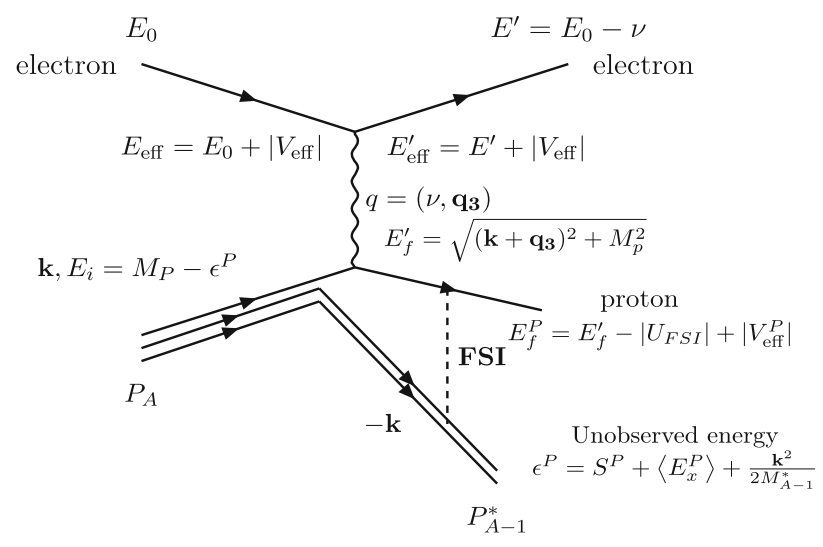

Fig. $31 \mathrm{p} 1 \mathrm{~h}$ process: Electron scattering from an off-shell bound proton of momentum $\boldsymbol{p}_{\boldsymbol{i}}=\boldsymbol{k}$ in a nucleus of mass A. Here, the nucleon is moving in the mean field (MF) of all the other nucleons in the nucleus. The on-shell recoil excited $[A-1]^{*}$ spectator nucleus has a momentum $\boldsymbol{p}_{(A-1) *}=-\boldsymbol{k}$ and a mean excitation energy $\left\langle E_{x}^{P}\right\rangle$. The off-shell energy of the interacting nucleon is $E_{i}=M_{A}-\sqrt{\left(M_{A-1} *\right)^{2}+\boldsymbol{k}^{2}}=M_{A}-$ $\sqrt{\left(M_{A-1}+E_{x}\right)^{2}+\boldsymbol{k}^{2}}=M_{P}-\epsilon^{P}$, where $\epsilon^{P}=S^{P}+\left\langle E_{x}\right\rangle+\frac{\boldsymbol{k}^{2}}{2 M_{A-1}^{*}}$. We model the effect of FSI (strong and EM interactions) by setting $E_{f}=$ $\sqrt{\left(\boldsymbol{k}+\boldsymbol{q}_{3}\right)^{2}+M_{P}^{2}}-\left|U_{F S I}\right|+\left|V_{e f f}^{P}\right|$, where $U_{F S I}=U_{F S I}\left(\left(\boldsymbol{q}_{3}+\boldsymbol{k}\right)^{2}\right)$. For electron QE scattering on bound protons $\left|V_{e f f}^{P}\right|=\frac{Z}{Z-1}\left|V_{e f f}\right|$, $E_{e f f}=E_{0}+V_{e f f}, E_{e f f}^{\prime}=E^{\prime}+V_{e f f}$ 
Table 1 The spin parity transitions and separation energies $S^{P}, S^{N}$ and $S^{N+P}$ when a proton or a neutron or both are removed from various nuclei. All energies are in $\mathrm{MeV}$

\begin{tabular}{|c|c|c|c|c|c|}
\hline${ }_{Z^{A} \mathrm{Nucl}}$ & $\begin{array}{l}\text { remove proton } \\
\text { Spectator }\end{array}$ & $S^{P}$ & $\begin{array}{l}\text { remove neutron } \\
\text { Spectator }\end{array}$ & $S^{N}$ & $\overline{S^{N+P}}$ \\
\hline${ }_{1}^{2} \mathrm{H}$ & $\mathrm{N}$ & 2.2 & $\mathrm{P}$ & 2.2 & 2.2 \\
\hline${ }_{3}^{6} \mathrm{Li} 1+$ & ${ }_{2}^{5} \mathrm{He} \frac{3}{2}-$ & 4.4 & ${ }_{3}^{5} \mathrm{Li} \frac{3}{2}-$ & 5.7 & 4.0 \\
\hline${ }_{6}^{12} \mathrm{C} 0+$ & ${ }_{5}^{11} \mathrm{~B} \frac{3}{2}-$ & 16.0 & ${ }_{6}^{11} \mathrm{C} \frac{3}{2}-$ & 18.7 & 27.4 \\
\hline${ }_{8}^{16} \mathrm{O} 0+$ & ${ }_{7}^{15} \mathrm{~N} \frac{1}{2}-$ & 12.1 & ${ }_{8}^{15} \mathrm{O} \frac{1}{2}-$ & 15.7 & 23.0 \\
\hline${ }_{12}^{24} \mathrm{Mg} 0+$ & ${ }_{11}^{23} N a \frac{3}{2}+$ & 11.7 & ${ }_{12}^{23} \mathrm{Mg} \frac{3}{2}+$ & 16.5 & 24.1 \\
\hline${ }_{13}^{27} \mathrm{Al} \frac{5}{2}+$ & ${ }_{12}^{26} \mathrm{Mg} \mathrm{0+}$ & 8.3 & ${ }_{12}^{23} \mathrm{Al} \mathrm{5+}$ & 13.1 & 19.4 \\
\hline${ }_{14}^{28} \mathrm{Si} \mathrm{0+}$ & ${ }_{13}^{27} \mathrm{Al} \frac{5}{2}+$ & 11.6 & ${ }_{14}^{27} \mathrm{Si} \frac{5}{2}+$ & 17.2 & 24.7 \\
\hline${ }_{18}^{40} \mathrm{Ar} \frac{3}{2}+$ & ${ }_{17}^{39} \mathrm{CL} \frac{3}{2}+$ & 12.5 & ${ }_{18}^{39} \mathrm{Ar} \frac{7}{2}-$ & 9.9 & 20.6 \\
\hline${ }_{20}^{40} \mathrm{Ca} 0+$ & ${ }_{19}^{39} \mathrm{~K} \frac{3}{2}+$ & 8.3 & ${ }_{20}^{39} \mathrm{Ca} \frac{3}{2}+$ & 15.6 & 21.4 \\
\hline${ }_{23}^{51} \mathrm{~V} \frac{7}{2}-$ & ${ }_{22}^{50} \mathrm{Ti} 0+$ & 8.1 & ${ }_{23}^{50} \mathrm{~V} 6+$ & 11.1 & 19.0 \\
\hline${ }_{26}^{56} \mathrm{Fe} 0+$ & ${ }_{25}^{55} \mathrm{Mn} \frac{5}{2}-$ & 10.2 & ${ }_{26}^{55} \mathrm{Fe} \frac{3}{2}-$ & 11.2 & 20.4 \\
\hline${ }_{28}^{58} \mathrm{Ni} \frac{3}{2}-$ & ${ }_{27}^{58} \mathrm{Co} 2+$ & 8.2 & ${ }_{87}^{58} \mathrm{Ni} 0+$ & 12.2 & 19.5 \\
\hline${ }_{39}^{89} \mathrm{Y} \frac{1}{2}-$ & ${ }_{38}^{88} \mathrm{Sr} \frac{1}{2}-$ & 7.1 & ${ }_{39}^{88} \mathrm{Y} 4-$ & 11.5 & 18.2 \\
\hline${ }_{40}^{90} \mathrm{Zr} \mathrm{0+}$ & ${ }_{39}^{89} \mathrm{Y} \frac{1}{2}-$ & 8.4 & ${ }_{40}^{88} \mathrm{Zr} \frac{9}{2}+$ & 12.0 & 17.8 \\
\hline${ }_{50}^{120} \mathrm{Sn} 0+$ & ${ }_{49}^{119} \operatorname{In} \frac{9}{2}+$ & 10.1 & ${ }_{50}^{119} \mathrm{Sn} \frac{1}{2}+$ & 8.5 & 17.3 \\
\hline${ }_{73}^{181} \mathrm{Ta} \frac{7}{2}-$ & ${ }_{72}^{180} \mathrm{Hf} 0+$ & 5.9 & ${ }_{73}^{180} \mathrm{Ta} 1+$ & 7.6 & 13.5 \\
\hline${ }_{79}^{197} \mathrm{Au} \frac{3}{2}+$ & ${ }_{78}^{196} \mathrm{Pt} \mathrm{0+}$ & 5.8 & ${ }_{79}^{196} \mathrm{Au} 2-$ & 8.1 & 13.7 \\
\hline${ }_{82}^{208} \mathrm{~Pb} 0+$ & ${ }_{81}^{207} \mathrm{TI} \frac{1}{2}+$ & 8.0 & ${ }_{82}^{207} \mathrm{~Pb} \frac{1}{2}-$ & 7.4 & 14.9 \\
\hline
\end{tabular}

$M_{A}-\sqrt{\left(M_{A-1}+E_{x}\right)^{2}+\boldsymbol{k}^{2}}=M_{P}-\epsilon^{P}$, where $\epsilon^{P}=$ $S^{P}+\left\langle E_{x}\right\rangle+\frac{\boldsymbol{k}^{2}}{2 M_{A-1}^{*}}$. As discussed in Sect. 4 we model the effect of FSI (strong and EM interactions) by setting $E_{f}=\sqrt{\left(\boldsymbol{k}+\boldsymbol{q}_{3}\right)^{2}+M_{P}^{2}}-\left|U_{F S I}\right|+\left|V_{e f f}^{P}\right|$, where $U_{F S I}=$ $U_{F S I}\left(\left(\boldsymbol{q}_{3}+\boldsymbol{k}\right)^{2}\right)$.

Table 1 shows the spin and parity of the initial state nucleus, and the spin parity of the ground state of the spectator nucleus when a bound proton or a bound neutron is removed via the $1 \mathrm{p} 1 \mathrm{~h}$ process.

The four-momentum transfer to the nuclear target is defined as $q=\left(\boldsymbol{q}_{3}, v\right)$. Here $\boldsymbol{q}_{3}^{2}$ is the 3-momentum transfer, $v$ is the energy transfer, and $Q^{2}=-q^{2}=\boldsymbol{q}^{2}-v^{2}$ is the square of the four-momentum transfer. For QE electron scattering on unbound protons (or neutrons) the energy transfer $v$ is equal to $Q^{2} / 2 M_{P, N}$ where $M_{P}$ is mass of the proton and $M_{N}$ is the mass of the neutron, respectively.

\subsection{Nuclear density corrections to $k_{F}^{P}$ and $k_{F}^{N}$}

The values of the Fermi momentum $k_{F}$ that are currently used in neutrino Monte Carlo generators are usually taken from an analysis of e-A data by Moniz et al. [17-19]. The Moniz published values of $k_{F}$ were extracted using the RFG model under the assumption that the Fermi momenta for protons and neutrons are different and are related to $k_{F}$ via the relations
$k_{F}^{N}=k_{F}(2 N / A)^{1 / 3}$ and $k_{F}^{P}=k_{F}(2 Z / A)^{1 / 3}$, respectively. What is actually measured is $k_{F}^{P}$, and what is published is $k_{F}$. Moniz assumes that the nuclear density (nucleons per unit volume) is constant. Therefore, in the same nuclear radius $\mathrm{R}$, $k_{F}^{N}$ for neutrons is larger if $\mathrm{N}$ is greater than $\mathrm{Z}$. Moniz used these expressions to extract the published value of $k_{F}$ from the measured value of $k_{F}^{P}$.

We undo this correction and re-extract the measured values of $k_{F}^{P}$ for nuclei which have a different number of neutrons and protons. In order to obtain the values of $k_{F}^{N}$ from the measured values of $k_{F}^{P}$ we use the fact that the Fermi momentum is proportional to the cube root of the nuclear density. Consequently $k_{F}^{N}=C \frac{N^{1 / 3}}{R_{N}}$, and $k_{F}^{P}=C \frac{Z^{1 / 3}}{R_{P}}$, and $k_{F}^{N}=k_{F}^{P} \frac{N^{1 / 3} R_{P}}{Z^{1 / 3} R_{N}}$. For the proton and neutron radii, we use the fits for the half density radii of nuclei (in units of femtometer) given in Ref. [29].

$$
\begin{aligned}
& R_{P}=1.322 Z^{1 / 3}+0.007 N+0.022 \\
& R_{N}=0.953 N^{1 / 3}+0.015 Z+0.774
\end{aligned}
$$

We only these fits for nuclei which do not have an equal number of protons and neutrons. For nuclei which have an equal number of neutrons and protons we assume that $k_{F}^{N}=$ $k_{F}^{P}=k_{F}($ Moniz $)$.

However for the ${ }_{82}^{208} \mathrm{~Pb}$ nucleus only we use $k_{F}^{P}=$ $0.275 \mathrm{GeV}$ which we obtain from our own fits to inclusive 


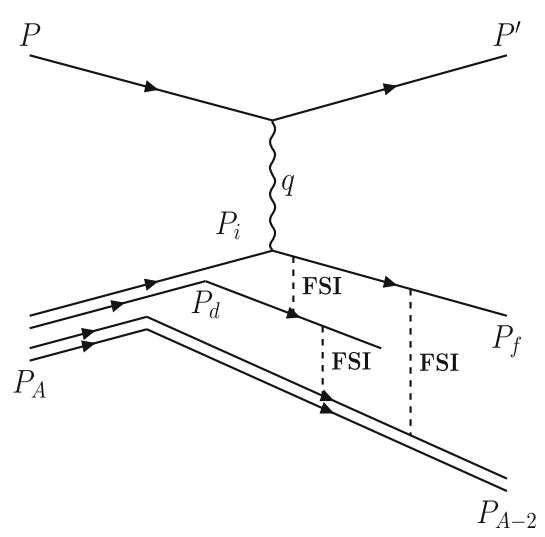

Fig. 4 2p2h process: Electron scattering from an off-shell bound proton of momentum $\boldsymbol{p}_{i}=\boldsymbol{k}$ from two nucleon short range correlations (quasi-deuteron). There is an on-shell spectator (A-2)* nucleus and an on-shell spectator recoil neutron with momentum $-\boldsymbol{k}$. The offshell energy of the interacting bound proton is $E_{i}^{P}(\operatorname{src})=M_{D}-$ $\sqrt{M_{N}+\boldsymbol{k}^{2}}-S^{P+N}$

e-A scattering data. For all other nuclei, our values are consistent with the values extracted by Moniz et al.

\subsection{Separation energy}

The separation energy for a proton $\left(S^{P}\right)$ or neutron $S^{N}$ is defined as follows:

$M_{A}=M_{A-1}+M_{N, P}-S^{N, P}$

Table 2 Summary of the relationships between excitation energy $E_{x}^{P, N}$ (used in GENIE), separation energy $S^{P, N}$, missing (missing) energy $E_{m}^{P, N}$ (used in spectral function measurements), removal energy $\epsilon^{P, N}$ (used in the reconstruction of neutrino energy from muon kinematics
The energy to separate both a proton and neutron $\left(S^{P+N}\right)$ is defined as follows:

$M_{A}=M_{A-2}+M_{P}+M_{N}-S^{N+P}$

The proton and neutron separation energies $\mathrm{S}^{P}$ and $\mathrm{S}^{N}$ are available in nuclear data tables. The values of $\mathrm{S}^{P}, \mathrm{~S}^{N}$ and $S^{N+P}$ for various nuclei $[30,31]$ are given in Table 1

\subsection{Two nucleon correlations}

Figure 4 illustrates the $2 \mathrm{p} 2 \mathrm{~h}$ process originating from both long range and short range two nucleon correlations (SRC). Here the scattering is from an off-shell bound proton of momentum $\boldsymbol{p}_{i}=\boldsymbol{k}$. The momentum of the initial state offshell interacting nucleon is balanced by a single on-shell correlated recoil neutron which has momentum $-\boldsymbol{k}$. The $[A-2]^{*}$ spectator nucleus is left with two holes. Short range nucleon-proton correlations occur $\approx 20 \%$ of the time [32]. The off-shell energy of the interacting bound proton in a quasi-deuteron is $\left(E_{i}^{P}\right)_{s r c}=M_{D}-\sqrt{M_{N}+\boldsymbol{k}^{2}}-S^{P+N}$, where $M_{D}$ is the mass of the deuteron. For QE scattering there is an additional $2 \mathrm{p} 2 \mathrm{~h}$ transverse cross section from "Meson Exchange Currents" (MEC) and "Isobar Excitation" (IE).

In this paper we only focus on the extraction of the average removal energy parameters for $1 \mathrm{p} 1 \mathrm{~h}$ processes. Processes leading to $2 \mathrm{p} 2 \mathrm{~h}$ final states (SRC, MEC and IE) result in larger missing energy and should be modeled separately.

only), the Smith-Moniz removal energy $\epsilon_{S M}^{\prime P, N}$ (that should be used in OLD- NEUT) and the parameters $x$ and $\left.x^{\nu, \bar{v}}\left(\boldsymbol{q}_{3}+\boldsymbol{k}\right)^{2}\right)$ which we use to include the effects of FSI in electron and neutrino/antineutrino scattering. For QE neutrino scattering on bound neutrons $\left|V_{e f f}^{P}\right|=\left|V_{e f f}\right|$

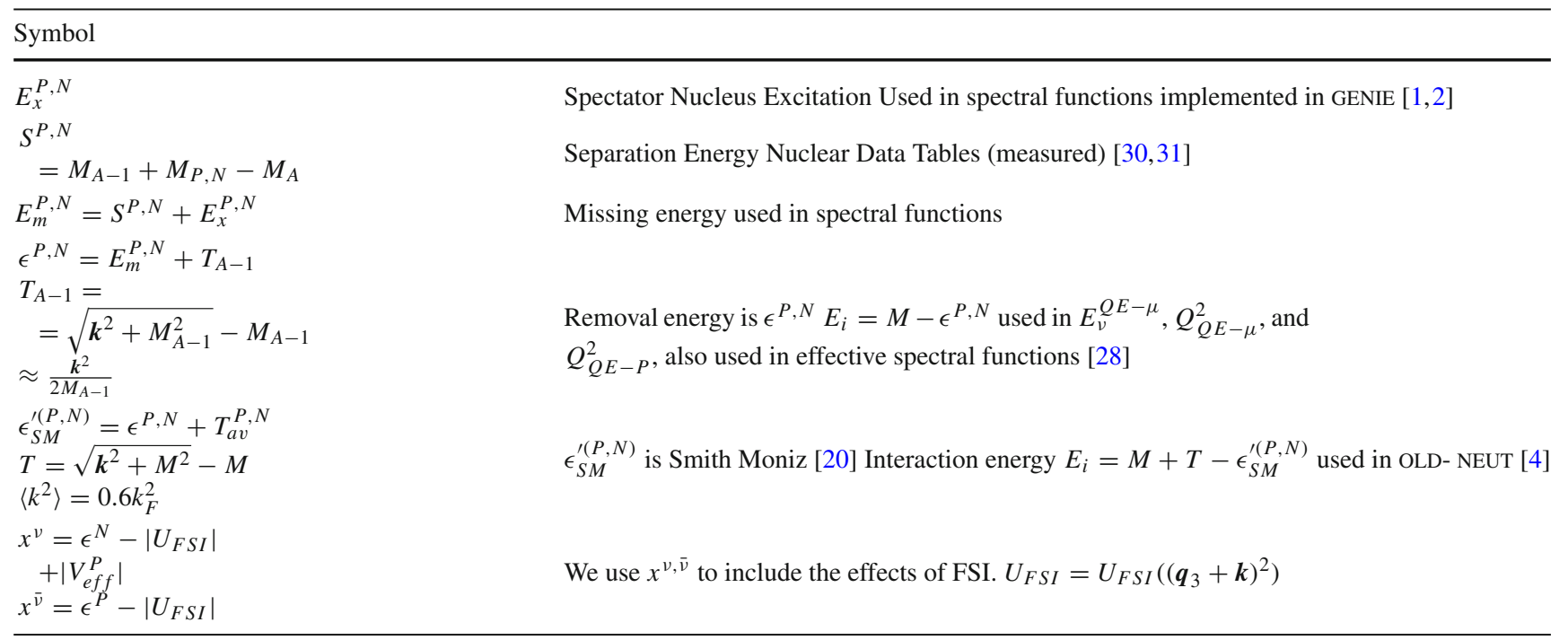


Table 3 Average values of the proton kinetic energy $\left\langle T^{P}\right\rangle^{S F}$ and missing energy $\left\langle E_{m}^{P}\right\rangle^{S F}$ for $1 \mathrm{p} 1 \mathrm{~h}$ final states $\left(E_{m}^{P}<80\right)$ extracted from published tests of the Koltun sum rule using spectral function (SF) measurements at Jefferson lab Hall A $[37,38]$ and Saclay $[39,40]$. For a Fermi gas distribution $\left\langle T^{P}\right\rangle=\frac{3}{5}\left(k_{F}^{P}\right)^{2}$ All energies are in $\mathrm{MeV}$. The bolded numbers are the best estimates for each target

\begin{tabular}{|c|c|c|c|}
\hline Target & Q2 & $\begin{array}{l}\left\langle T^{P}\right\rangle \\
E_{m}^{P}<80\end{array}$ & $\begin{array}{l}\left\langle E_{m}^{P}\right\rangle \\
E_{m}^{P}<80\end{array}$ \\
\hline${ }_{6}^{12} \mathrm{C}$ & 0.6 & 15.9 & 26.0 \\
\hline \multirow[t]{3}{*}{ Jlab Hall C $[37,38]$} & 1.2 & 16.3 & 25.8 \\
\hline & 1.8 & 16.0 & 26.6 \\
\hline & 3.2 & 17.3 & 26.2 \\
\hline $\mathrm{Jlab}\left\langle T^{P}\right\rangle^{S F},\left\langle E_{m}^{P}\right\rangle^{S F}$ & Ave. & $16.4 \pm 0.6$ & $26.1 \pm 0.4$ \\
\hline Saclay $\left\langle T^{P}\right\rangle^{S F},\left\langle E_{m}^{P}\right\rangle^{S F}$ & & $16.9 \pm 0.5$ & $23.4 \pm 0.5$ \\
\hline Saclay $\left\langle E_{m}^{P}\right\rangle^{\text {levels }}$ & & & $24.4 \pm 2$ \\
\hline$k_{F}^{P}=221 \pm 5$ & & $15.5 \pm 1.2$ & \\
\hline Target & Q2 & $\left\langle T^{P}\right\rangle$ & $\left\langle E_{m}^{P}\right\rangle$ \\
\hline \multicolumn{4}{|l|}{${ }_{14}^{28} \mathrm{Si}$} \\
\hline Saclay $\left\langle T^{P}\right\rangle^{S F},\left\langle E_{m}^{P}\right\rangle^{S F}$ & & $17.0 \pm 0.6$ & $24.0 \pm 0.6$ \\
\hline Saclay $\left\langle E_{m}^{P}\right\rangle^{\text {levels }}$ & & & $27.6 \pm 2$ \\
\hline$k_{F}^{P}=239 \pm 5$ & & $18.1 \pm 1.3$ & \\
\hline Target & Q2 & $\left\langle T^{P}\right\rangle$ & $\left\langle E_{m}^{P}\right\rangle$ \\
\hline \multicolumn{4}{|l|}{${ }_{20}^{40} \mathrm{Ca}$} \\
\hline Saclay $\left\langle T^{P}\right\rangle^{S F},\left\langle E_{m}^{P}\right\rangle^{S F}$ & & $16.6 \pm 0.5$ & $27.8 \pm 0.5$ \\
\hline $\operatorname{Saclay}\left\langle E_{m}^{P}\right\rangle^{\text {levels }}$ & & & $26.5 \pm 2$ \\
\hline$k_{F}^{P}=239 \pm 5$ & & $18.1 \pm 1.3$ & \\
\hline Target & Q2 & $\left\langle T^{P}\right\rangle$ & $\left\langle E_{m}^{P}\right\rangle$ \\
\hline${ }_{26}^{56} \mathrm{Fe}$ & 0.6 & 20.4 & 30.7 \\
\hline \multirow[t]{3}{*}{ Jlab Hall C $[37,38]$} & 1.2 & 18.1 & 29.4 \\
\hline & 1.8 & 17.8 & 27.8 \\
\hline & 3.2 & 19.1 & 28.8 \\
\hline $\mathrm{Jlab}\left\langle T^{P}\right\rangle^{S F},\left\langle E_{m}^{P}\right\rangle^{S F}$ & Ave. & $18.8 \pm 1.0$ & $29.2 \pm 1.1$ \\
\hline$k_{F}^{P}=254 \pm 5$ & & $20.4 \pm 1.4$ & \\
\hline Target & Q2 & $\left\langle T^{P}\right\rangle$ & $\left\langle E_{m}^{P}\right\rangle$ \\
\hline \multicolumn{4}{|l|}{${ }_{28}^{58} \mathrm{Ni}$} \\
\hline Saclay $\left\langle T^{P}\right\rangle^{S F},\left\langle E_{m}^{P}\right\rangle^{S F}$ & & $18.8 \pm 0.7$ & $25.0 \pm 0.7$ \\
\hline $\operatorname{Saclay}\left\langle E_{m}^{P}\right\rangle^{l \text { evels }}$ & & & $25.3 \pm 2$ \\
\hline$k_{F}^{P}=257 \pm 5$ & & $20.9 \pm 1.4$ & \\
\hline Target & Q2 & $\left\langle T^{P}\right\rangle$ & $\left\langle E_{m}^{P}\right\rangle$ \\
\hline${ }_{79}^{197} \mathrm{Au}$ & 0.6 & 20.2 & 25.5 \\
\hline \multirow[t]{3}{*}{ Jlab Hall C [37,38] } & 1.2 & 18.4 & 25.7 \\
\hline & 1.8 & 18.3 & 24.1 \\
\hline & 3.2 & 19.4 & 26.1 \\
\hline $\mathrm{Jlab}\left\langle T^{P}\right\rangle^{S F},\left\langle E_{m}^{P}\right\rangle^{S F}$ & Ave. & $19.1 \pm 0.8$ & $25.3 \pm 0.8$ \\
\hline$k_{F}^{P}=24.5 \pm 5$ & & $19.0 \pm 1.3$ & \\
\hline
\end{tabular}

\section{Spectral functions and $e e^{\prime} p$ experiments}

In $e e^{\prime} p$ experiments the following process is investigated:

$e+A \rightarrow e^{\prime}+(A-1)^{\star}+p_{f}$.

Here, an electron beam is incident on a nuclear target of mass $M_{A}$. The hadronic final state consists of a proton of four momentum $p_{f} \equiv\left(E_{f}, \boldsymbol{p}_{f}\right)$ and an undetected nuclear remnant $(A-1)^{\star}$. Both the final state electron and the final state proton are measured. The $(A-1)^{\star}$ nuclear remnant can be a $(A-1, Z-1)$ spectator nucleus with excitation $E_{x}^{P}$, or a nuclear remnant with additional unbound nucleons.

At high energies, within the plane wave impulse approximation (PWIA) the initial momentum $\boldsymbol{k}$ of the initial state off-shell interacting nucleon can be identified approximately with the missing momentum $\boldsymbol{p}_{m}$. Here we define $p_{m}=\left|\boldsymbol{p}_{m}\right|$ and $k=|\boldsymbol{k}|$

$\boldsymbol{p}_{m}=\boldsymbol{p}_{f}-\boldsymbol{q}_{3} \approx \boldsymbol{k}$

The missing energy $E_{m}$ is defined by the following relativistic energy conservation expression,

$$
\begin{aligned}
& v+M_{A}=\sqrt{\left(M_{A-1}^{*}\right)^{2}+\boldsymbol{p}_{\boldsymbol{m}}^{2}}+E_{f}^{P} \\
& E_{f}^{P}=\sqrt{\boldsymbol{p}_{f}^{2}+M_{P}^{2}}, \quad M_{A-1}^{*}=M_{A}-M+E_{m} .
\end{aligned}
$$

The missing energy $E_{m}$ can be expressed in term of the excitation energy $\left(E_{x}\right)$ of the spectator (A-1) nucleus and the separation energy of the proton $S^{P}$ (or neutron $S^{N}$ ).

$E_{m}^{P, N}=S^{P, N}+E_{x}^{P, N}$

The probability distribution of finding a nucleon with initial state momentum $p_{m} \approx k$ and missing energy $E_{m}$ from the target nucleus is described by the spectral function, defined as $P_{S F}\left(p_{m}, E_{m}\right)$. Note that for spectral functions both $P\left(p_{m}, E_{m}\right)$ and $S\left(p_{m}, E_{m}\right)$ notation are used in some publications. The spectral functions $P_{S F}^{P}\left(p_{m}, E_{m}^{P}\right)$ and $P_{S F}^{N}\left(p_{m}, E_{m}^{N}\right)$ for protons and neutrons are two dimensional distributions which can be measured (or calculated theoretically). Corrections for final state interactions of the outgoing nucleon are required in the extraction of $P_{S F}^{P}\left(p_{m}, E_{m}^{P}\right)$ from $e e^{\prime} p$ data. The kinematical region corresponding to low missing momentum and energy is where shell model [33] states dominate [34]. In practice, only the spectral function for protons can be measured reliably.

In addition to the $1 \mathrm{p} 1 \mathrm{~h}$ contribution in which the residual nucleus is left in the ground or excited bound state, the measured spectral function includes contributions from nucleonnucleon correlations in the initial state $(2 \mathrm{p} 2 \mathrm{~h})$ where there is one or more additional spectator nucleons. Spectral function measurements cannot differentiate between a spectator (A-1) 
nucleus and a spectator (A-2) nucleus from SRC because the 2nd final state SRC spectator nucleon is not detected.

Here, we focus on the spectral function for the $1 \mathrm{p} 1 \mathrm{~h}$ process, which dominates for $E_{m}$ less than $80 \mathrm{MeV}$, and ignore the spectral function for the $2 \mathrm{p} 2 \mathrm{~h}$ process which dominates at higher values of $E_{m}$. We use shell model calculations to obtain the difference in the binding energy parameters for neutrons and protons.

\section{Effects of the optical and Coulomb potentials (FSI)}

We use empirical parameter $U_{F S I}\left(\left(\boldsymbol{q}_{3}+\boldsymbol{k}\right)^{2}\right)$ to approximate the effect of the interaction of the final state proton with the optical potential of the spectator nucleus. This is important at low values of $\boldsymbol{q}_{3}^{2}$. In addition, we include the effect of the interaction of the final state proton with the Coulomb field of the nucleus $\left(V_{e f f}^{P}\right)$.

In QE scattering of electrons a three momentum transfer $\boldsymbol{q}_{3}$ to a bound proton with initial momentum $\boldsymbol{k}$ results in the following energy $E_{f}^{P}$ of the final state proton:

$E_{f}^{P}=\sqrt{\left(k+\boldsymbol{q}_{3}\right)^{2}+M_{P}^{2}}-\left|U_{F S I}\left(\left(\boldsymbol{q}_{3}+\boldsymbol{k}\right)^{2}\right)\right|+\left|V_{e f f}^{P}\right|$

where for electron scattering on bound protons $V_{e f f}^{P}=$ $\frac{Z-1}{Z}\left|V_{e f f}\right|$. The Coulomb correction $\left|V_{e f f}\right|$ is discussed in Appendix A.

We define the average removal energy $\epsilon^{P, N}$ in terms of the average momentum $\left\langle k^{2}\right\rangle^{P, N}$ of the bound nucleon as follows:

$$
\begin{aligned}
\epsilon^{P, N} & =E_{m}^{P, N}+T_{A-1}^{N, P} \\
& =S^{P, N}+E_{x}^{P, N}+\frac{\left\langle k^{2}\right\rangle^{P, N}}{2 M_{A-1}^{*}}
\end{aligned}
$$

In order to properly simulate neutrino interactions we extract values of the average missing energy (or equivalently the average excitation energy) from spectral functions measured in ee'p experiments. We then use these values and extract $U_{F S I}\left(\left(\boldsymbol{q}_{3}+\boldsymbol{k}\right)^{2}\right)$ from inclusive e-A as discussed in Sect. 9.

\subsection{Smith-Moniz formalism}

The Smith-Moniz [20] formalism uses on-shell description of the initial state. In the on-shell formalism, the energy conserving expression is

$\nu+M-\epsilon=E_{f}$

is replaced with

$v+\sqrt{k^{2}+M^{2}}-\epsilon_{S M}^{\prime P, N}=E_{f}$.

Therefore,

$\epsilon_{S M}^{\prime}=\epsilon+\left\langle T^{P, N}\right\rangle$,

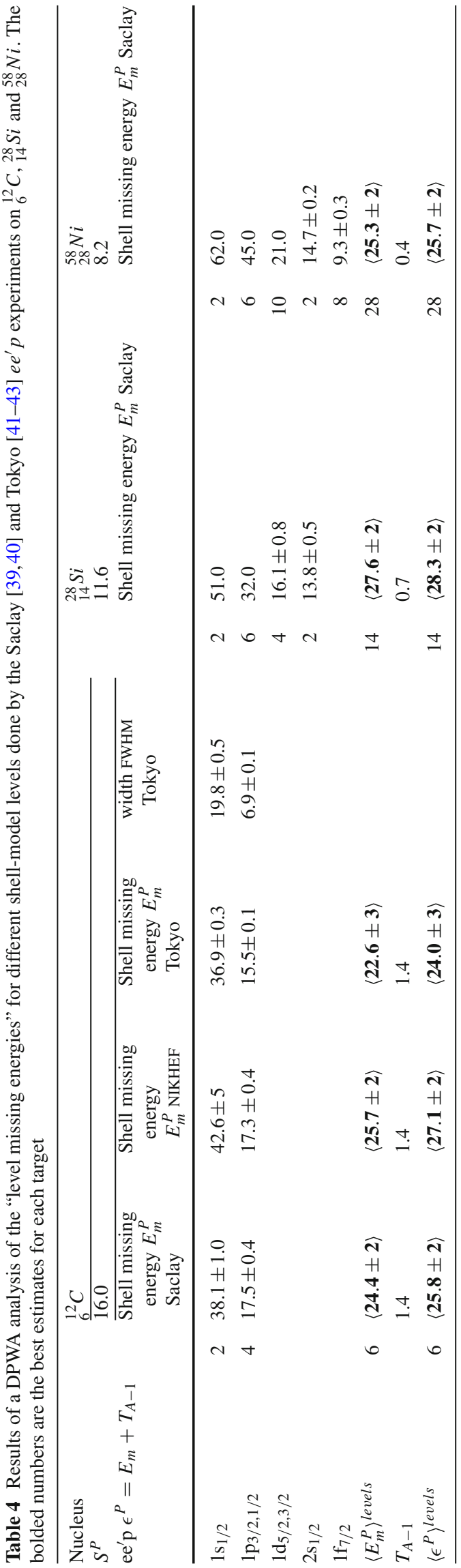




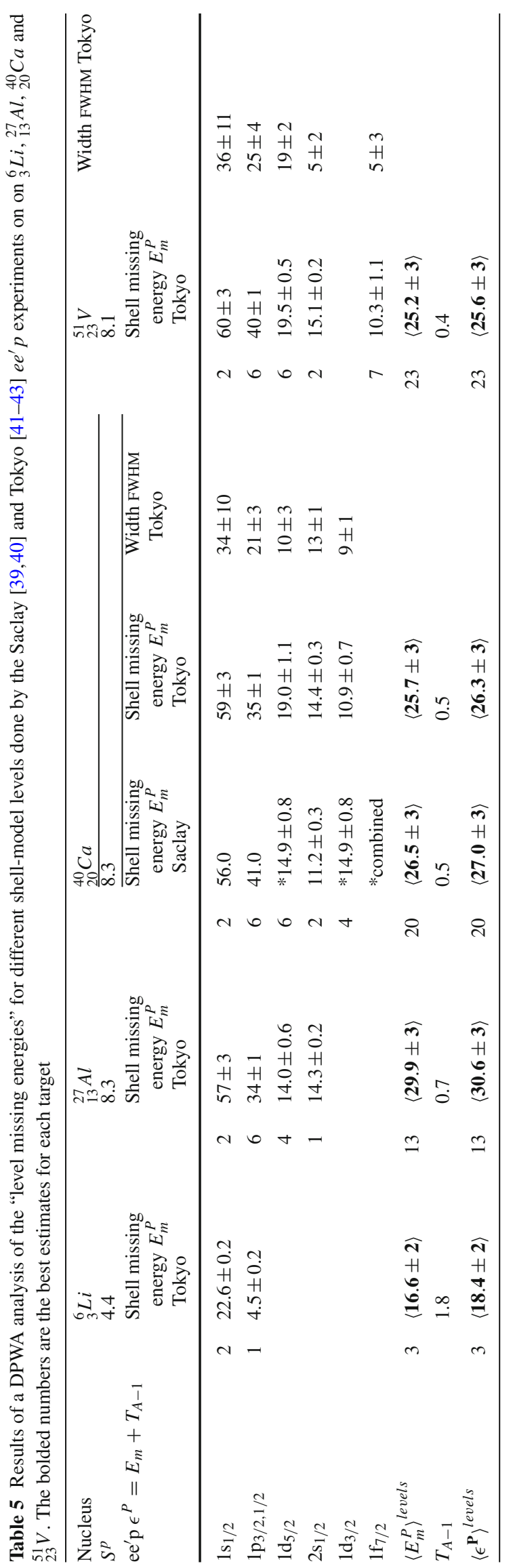

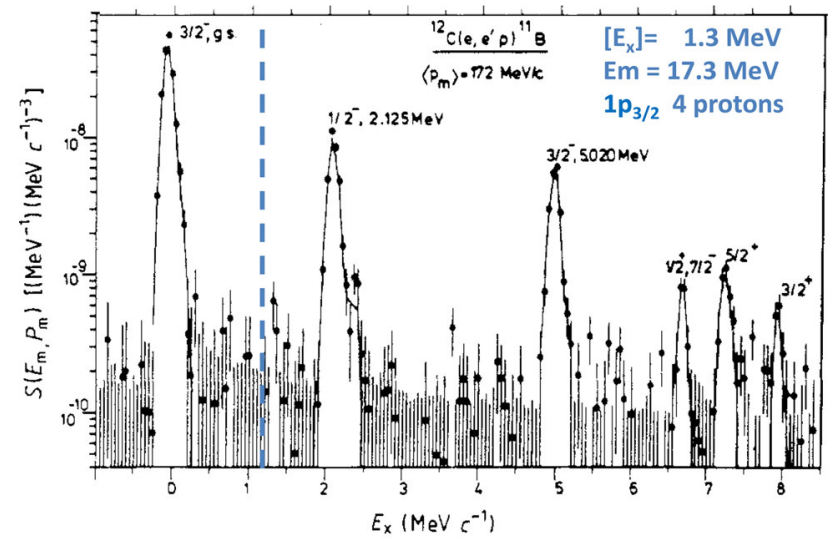

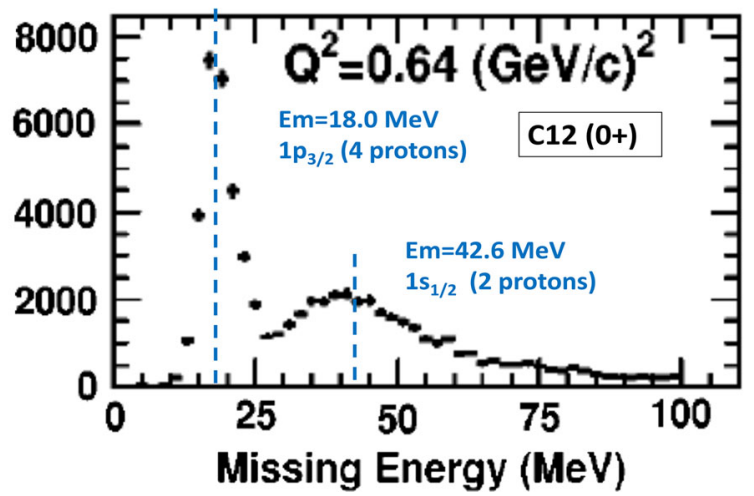

Fig. 5 Top panel: The measured [44,45] NIKHEF high resolution spectral function for protons in the $1 \mathrm{p}$ level of ${ }_{6}^{12} \mathrm{C}$ as a function of the spectator nucleus excitation energy $E_{x}^{P}$ for $\boldsymbol{p}_{m}=\boldsymbol{k}=172 \mathrm{MeV} / \mathrm{c}$. Bottom panel: The Jlab measurement $[37,38]$ of the one-dimensional spectral function for ${ }_{6}^{12} C$ as a function of missing energy $E_{m}^{P}$ for $Q^{2}=0.64 \mathrm{GeV}^{2}$

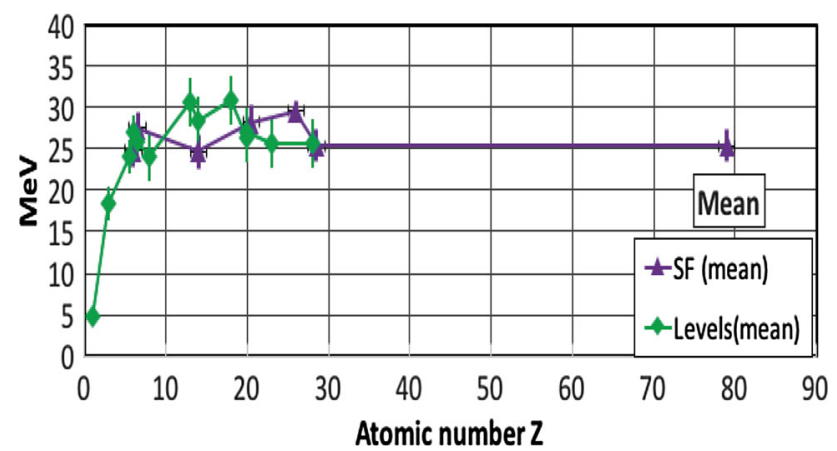

Fig. 6 Average removal energies versus atomic number Z. Values of $\left\langle\epsilon^{P}\right\rangle^{S F}$ from tests of the Koltun sum rule in $e e^{\prime} p$ experiments (in purple) are compared to values of $\left\langle\epsilon^{P}\right\rangle^{\text {levels }}$ extracted from $e e^{\prime} p$ measurements of "level missing energies" (in green)

where

$\left\langle T^{P, N}\right\rangle=\sqrt{\left\langle k^{2}\right\rangle^{P, N}+M^{2}}-M \approx \frac{3}{5} \frac{\left(k_{F}^{P, N}\right)^{2}}{2 M}$

A summary of the relationships between excitation energy $E_{x}^{P, N}$ used in GENIE (which incorporates the Bodek-Ritchie [35] off-shell formalism), separation energy $S^{P, N}$, missing 


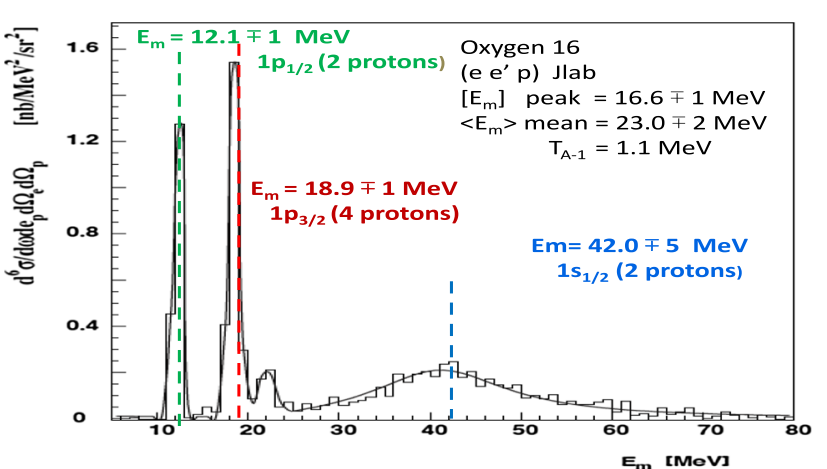

Fig. 7 The spectral function for ${ }_{8}^{16} O$ as a function of $E_{m}^{P}$ for $\boldsymbol{p}_{m}=$ $\boldsymbol{k}=60 \mathrm{MeV} / \mathrm{c}$ measured in Jlab Hall A [48-50] with $2.4 \mathrm{GeV}$ incident electrons

missing energy $E_{m}^{P, N}$ (used in spectral function measurements), removal energy $\epsilon^{P, N}$ (used in the reconstruction of neutrino energy from muon kinematics only) and the Smith-Moniz removal energy $\epsilon_{S M}^{\prime P, N}$ (that should be used in OLD- NEUT) is given in Table 2.

\section{Extraction of average missing energy $\left\langle E_{m}\right\rangle$}

We extract the average missing energy $\left\langle E_{m}^{P}\right\rangle$ and excitation energy $\left\langle E_{x}^{P}\right\rangle$ for the $1 \mathrm{p} 1 \mathrm{~h}$ process from $e e^{\prime} P$ electron scattering data using two methods.

1. $\left\langle E_{m}^{P}\right\rangle^{S F}$ : From direct measurements of the average missing energy $\left\langle E_{m}^{P}\right\rangle$ and average proton kinetic energy $\left\langle T^{P}\right\rangle$. These quantities have been extracted from spectral functions measured in ee' $\mathrm{p}$ experiments for tests of the Koltun sum rule [36]. The contribution of two nucleon corrections is minimized by restricting the analysis to $E_{m}^{P}<$ $80 \mathrm{MeV}$. This is the most reliable determination of $\left\langle E_{m}^{P}\right\rangle$. We refer to this average as $\left\langle E_{m}^{P}\right\rangle^{S F}$.

2. $\left\langle E_{m}^{P}\right\rangle^{\text {levels }}$ : By taking the average (weighted by shell model number of nucleons) of the nucleon "level missing energies" of all shell model levels which are extracted from spectral functions measured in ee'p experiments. We refer to this average as $\left\langle E_{m}^{P}\right\rangle^{\text {levels }}$.

There could be bias in method 2 originating from the fact that a fraction of the nucleons $(\approx 20 \%)$ in each level are in a correlated state with other nucleons (leading to $2 \mathrm{p} 2 \mathrm{~h}$ final states). The fraction of correlated nucleons is not necessarily the same for all shell-model levels. As discussed in Sect. 5.4 (and shown Fig. 6) we find that the values of $\left\langle E_{m}^{P}\right\rangle^{\text {levels }}$ are consistent with $\left\langle E_{m}^{P}\right\rangle^{S F}$ for nuclei for which both are available.

When available, we extract the removal energy parameters using $\left\langle E_{m}^{P}\right\rangle^{S F}$ from method 1. Otherwise we use $\left\langle E_{m}^{P}\right\rangle^{\text {levels }}$ from method 2. For each of the two methods, we also use

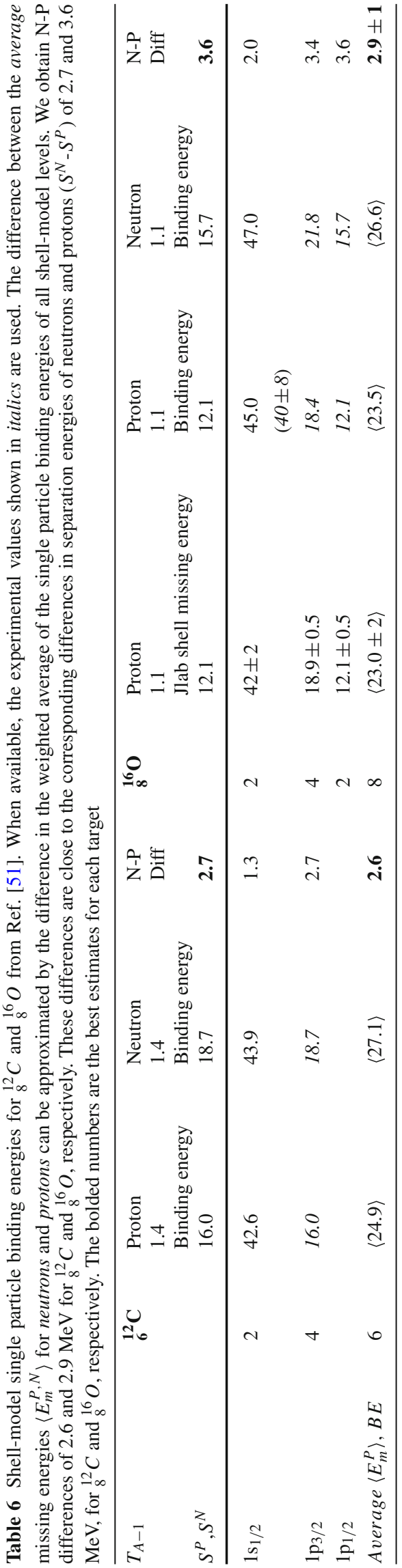


the nuclear shell model to estimate difference between the missing energies for neutrons and protons.

\subsection{Direct measurements of $\left\langle E_{m}^{P}\right\rangle^{S F}$ and $\left\langle T^{P}\right\rangle^{S F}$}

The best estimates of the average missing energy $\left\langle E_{m}^{P}\right\rangle$ and average nucleon kinetic energy $\left\langle T^{P}\right\rangle$ are those that are directly extracted from spectral function measurements in analyses that test the Koltun sum rule [36]. The Koltun's sum rule states that

$\frac{E_{B}}{A}=\frac{1}{2}\left[\left\langle T^{P}\right\rangle^{S F} \frac{A-2}{A-1}-\left\langle E_{m}^{P}\right\rangle^{S F}\right]$,

where $E_{B} / A$ is the nuclear binding energy per particle obtained from nuclear masses and includes a (small) correction for the Coulomb energy,

$\left\langle T^{P}\right\rangle^{S F}=\int d^{3} k d E_{m} \frac{\boldsymbol{k}^{2}}{2 M} P_{S F}\left(k, E_{m}\right)$,

and

$\left\langle E_{m}\right\rangle^{S F}=\int d^{3} k d E_{m} E_{m} P_{S F}\left(k, E_{m}\right)$.

For precise tests of the Koltun sum rule a small contribution from three-nucleon processes should be taken into account.

Values of $\left\langle E_{m}^{P}\right\rangle^{S F}$ and $\left\langle T^{P}\right\rangle^{S F}$ for the $1 \mathrm{p} 1 \mathrm{~h}$ process $\left(E_{m}^{P}<80 \mathrm{MeV}\right)$ published by Jlab Hall $\mathrm{C}$ experiments $[37,38]$ and by the Saclay group $[39,40]$ are given in Table 3.

\subsection{Spectral function "level missing energies"}

Measured 2D spectral functions can be analyzed within the distorted plane wave approximation (DPWA) to extract the peak and width of the missing energy distribution $E_{m}^{P}$ for protons for each shell model level. We refer to it as the "level missing energy". In some publications it is referred to as the "shell separation energy". The energies and widths of the "level missing energies" for ${ }_{3}^{6} \mathrm{Li},{ }_{6}^{12} \mathrm{C},{ }_{3}^{17} \mathrm{Al},{ }_{20}^{40} \mathrm{Ca},{ }_{23}^{50} \mathrm{~V}$, extracted from data published by the Tokyo group [41-43] are shown in Tables 4 and 5. Also shown are the "level missing energies" for ${ }_{6}^{12} \mathrm{C},{ }_{14}^{28} \mathrm{Si},{ }_{20}^{40} \mathrm{Ca}$, and ${ }_{28}^{58.7} \mathrm{Ni}$, extracted from the data published by the Saclay $[39,40]$ group.

We obtain an estimate of the average missing energy $\left\langle E_{m}^{P}\right\rangle^{\text {levels }}$ for the $1 \mathrm{p} 1 \mathrm{~h}$ process by taking the average (weighted by the number of nucleons) of the "level missing energies" of all shell model levels with $E_{m}^{P}<80 \mathrm{MeV}$. The results of our analysis of the Saclay and Tokyo data are given in Tables 4 and 5. As shown in Tables 4 and 5 for the deeply bound $\mathbf{1 s}$ and $\mathbf{1 p}$ levels in heavy nuclei the averages and widths of the missing energy distributions are large.

\section{$5.3{ }_{6}^{12} C$ spectral function}

The measured [44-47] NIKHEF high resolution spectral function for the missing energy of a bound proton in the $1 \mathrm{p}$ level of ${ }_{6}^{12} C$ as a function of the spectator nucleus excitation energy $E_{x}^{P}$ for $\boldsymbol{p}_{m}=\boldsymbol{k}=172 \mathrm{MeV} / \mathrm{c}$ is shown in the top panel of Fig. 5. The Jlab measurement [37,38] of the one-dimensional spectral function for the missing energy for bound proton from ${ }_{6}^{12} C$ as a function of $E_{m}^{P}$ for $Q^{2}=0.64$ $\mathrm{GeV}^{2}$ is shown in the bottom panel of of Fig. 5. The second peak at an average value of $E_{m}^{P} \approx 42.6 \pm 5 \mathrm{MeV}$ is for protons in the $1 \mathrm{~s}$ level. Combining the two results (weighted by the number of nucleons in each level) we obtain $\left\langle E_{m}^{P}\right\rangle^{\text {levels }}=25.7$ $\pm 2 \mathrm{MeV}$ for ${ }_{6}^{12} C$. Additional details are given in Table 4 .

\subsection{Comparison of the two methods}

Tests of the Koltun sum rule as a function of $Q^{2}$ were done by $e e^{\prime} p$ experiments at Jlab Hall C $[37,38]$ for ${ }_{6}^{12} C,{ }_{26}^{56} F e$, and ${ }_{79}^{197} \mathrm{Au}$. Tests of the Koltun sum rule were also reported by the Saclay [39,40] group for ${ }_{6}^{12} \mathrm{C},{ }_{14}^{28} \mathrm{Si},{ }_{20}^{40} \mathrm{Ca}$, and ${ }_{28}^{59} \mathrm{Ni}$. For both groups values of $\left\langle E_{m}^{P}\right\rangle^{S F}$ and $\left\langle T^{P}\right\rangle^{S F}$ were extracted from the measured spectral functions. The results from both groups are summarized in Table 3. We take the RMS variation with $Q^{2}$ of the Jefferson Lab Hall C data shown in Table $3(\approx 0.5$ $\mathrm{MeV})$ as the random error in the Jlab Hall $\mathrm{C}$ measurements of $\left\langle E_{m}^{P}\right\rangle^{S F}$.

We use the $2.7 \mathrm{MeV}$ difference in the measured values of $\left\langle E_{m}^{P}\right\rangle^{S F}$ for ${ }_{6}^{12} C$ at Jefferson Lab (26.1 \pm 0.4$)$ and Saclay $(23.4 \pm 0.5)$ as the systematic error in measurements of $\left\langle E_{m}^{P}\right\rangle^{S F}$. Since $\left\langle E_{m}^{P}\right\rangle^{S F}$ is the most reliable measurement of $\left\langle E_{m}^{P}\right\rangle$, we assign $\pm 3 \mathrm{MeV}$ as the systematic uncertainty to all measurements of $\left\langle E_{m}^{P}\right\rangle$.

The average values of the removal energies $\left\langle\epsilon^{P}\right\rangle^{S F}=$ $\left\langle E_{m}^{P}\right\rangle^{S F}+T_{A-1}$ versus atomic number from tests of the Koltun sum rule are in agreement with the average values extracted from measurements of "level missing energies" $\left\langle\epsilon^{P}\right\rangle^{\text {levels }}=\left\langle E_{m}^{P}\right\rangle^{\text {levels }}+T_{A-1}$ as shown Fig. 6. For example, for the Saclay data shown in Table 3 the average of the difference between $\left\langle E_{m}^{P}\right\rangle^{\text {levels }}$ and $\left\langle E_{m}^{P}\right\rangle^{S F}$ for ${ }_{6}^{12} C,{ }_{14}^{28} S i$, ${ }_{20}^{40} \mathrm{Ca}$, and ${ }_{28}^{59} \mathrm{Ni}$ is $0.9 \pm 1.0 \mathrm{MeV}$.

\subsection{Spectral function measurement of ${ }_{8}^{16} \mathrm{O}$}

The spectral function for ${ }_{8}^{16} O$ as a function of $E_{m}^{P}$ for $\boldsymbol{p}_{m}=$ $\boldsymbol{k}=60 \mathrm{MeV} / \mathrm{c}$ measured in Jlab Hall A [48-50] with 2.4 $\mathrm{GeV}$ incident electrons is shown in the top panel of Fig. 7. From this figure we extract a average $\left\langle E_{m}^{P}\right\rangle^{\text {levels }}=23.0 \pm$ $2 \mathrm{MeV}$.

Using $T_{A-1}=1.1 \mathrm{MeV}$ for ${ }_{8}^{16} O$ we also obtain a average $\left\langle\epsilon^{P}\right\rangle^{\text {levels }}=24.1 \pm 2 \mathrm{MeV}$. 
5.6 Difference between neutrons and protons for ${ }_{8}^{12} C$ and ${ }_{8}^{16} \mathrm{O}$

For nuclei which have the same number of neutrons and protons we expect that the average excitation energy $E_{x}^{P, N}$ spectrum for protons and neutrons to be approximately the same $\left(\left\langle E_{x}^{P, N}\right\rangle \approx\left\langle E_{x}^{P, N}\right\rangle\right)$. Since $E_{m}^{P, N}=S^{P, N}+E_{x}^{P, N}$ the difference in the average missing energies for neutrons and protons is approximately equal to the difference in separation energies $S^{N}-S^{P}$. By definition, the single particle binding energy of the least bound state is equal to the separation energy. The differences in the separation energies between neutrons and protons $\left(\mathrm{S}^{\mathrm{N}}-\mathrm{S}^{P}\right)$ bound in ${ }_{8}^{12} \mathrm{C}$ and ${ }_{8}^{16} \mathrm{O}$ are of $2.7 \mathrm{MeV}$ and $3.6 \mathrm{MeV}$, respectively.

More generally, a better estimate of the difference between the average missing energies for neutrons and protons can be obtained from the nuclear shell model. The single nucleon missing energy $\left(E_{m}^{P, N}\right)^{\text {shell-model-level }}$ for a nucleon in a given shell-model level is close (somewhat larger) to the single nucleon binding energy for that level. Consequently, the difference in the average missing energies for neutrons and protons for a nucleus $\left\langle E_{m}^{P}\right\rangle-\left\langle E_{m}^{N}\right\rangle$ is also approximately equal to the difference in the average binding energies.

The binding energies of different shell-model levels [51] for ${ }_{8}^{12} \mathrm{C}$ and ${ }_{8}^{16} \mathrm{O}$ are shown in Table 6. When available, the experimental values shown in italics are used.

The differences between the averages of the nucleon binding energies in all shell-model levels for neutrons and protons is 2.6 and $2.9 \mathrm{MeV}$ for ${ }_{8}^{12} \mathrm{C}$ and ${ }_{8}^{16} \mathrm{O}$, respectively. As expected these values are similar (within $1 \mathrm{MeV}$ ) to the differences in the separation energies for neutrons and protons $\left(\mathrm{S}^{\mathrm{N}}-\mathrm{S}^{P}\right)$ bound in ${ }_{8}^{12} \mathrm{C}$ and ${ }_{8}^{16} \mathrm{O}$ of of 2.7 and $3.6 \mathrm{MeV}$, respectively.

\section{Inclusive e-A electron scattering}

For QE electron scattering at low $\left(\boldsymbol{k}+\boldsymbol{q}_{3}\right)^{2}$ we use an empirical parameter $U_{F S I}\left(\left(\boldsymbol{k}+\boldsymbol{q}_{3}\right)^{2}\right)$ to account for the effect of final state interactions. The off-shell Bodek-Ritchie formalism (used by GENIE) for the case of QE electron scattering from a bound proton, should be implemented as follows:

$$
\begin{gathered}
v+\left(M_{P}-\epsilon^{P}\right)=\sqrt{\left(\mathbf{k}+\boldsymbol{q}_{\mathbf{3}}\right)^{\mathbf{2}}+M_{P}^{2}}-\left|U_{F S I}\right|+\left|V_{\text {eff }}^{P}\right| \\
\epsilon^{P}=S^{P}+\left\langle E_{x}^{P}\right\rangle+\frac{\left\langle\boldsymbol{k}^{2}\right\rangle}{2 M_{A-1}^{*}} \\
v+\left(M_{P}-x^{P}\right)=\sqrt{\left(\mathbf{k}+\boldsymbol{q}_{3}\right)^{2}+M_{P}^{2}} \\
x^{P}=S^{P}+\left\langle E_{x}^{P}\right\rangle+\frac{\left\langle k^{2}\right\rangle}{2 M_{A-1}}-\left|U_{F S I}\right|+\left|V_{e f f}^{P}\right| \\
x^{N}=S^{N}+\left\langle E_{x}^{N}\right\rangle+\frac{\left\langle k^{2}\right\rangle}{2 M_{A-1}}-\left|U_{F S I}\right|
\end{gathered}
$$

$$
\begin{aligned}
& Q^{2}=4\left(E_{0}+\left|V_{e f f}\right|\right)\left(E^{\prime}+\mid V_{e f f}\right) \mid \sin ^{2}(\theta / 2) \\
& E^{\prime}=E_{0}-v, \quad E_{f}^{P}=v-\epsilon^{P}, \quad \boldsymbol{q}_{3}^{2}=Q^{2}+v^{2},
\end{aligned}
$$

and $U_{F S I}=U_{F S I}\left(\left(\boldsymbol{q}_{3}+\boldsymbol{k}\right)^{2}\right)$. For electron scattering from a bound proton $\left|V_{e f f}^{P}\right|=\frac{Z-1}{Z}\left|V_{\text {eff }}\right|$, where $(\mathrm{Z}-1)$ is the number of protons in the spectator final state nucleus.

\subsection{Smith-Moniz on-shell formalism}

For QE electron scattering on a bound proton in the SmithMoniz on-shell formalism (used by OLD- NEUT) the following equations should be used:

$$
\begin{aligned}
& v+M_{P}+T^{P}-\epsilon_{S M}^{\prime P} \\
& =\sqrt{\left(k+\boldsymbol{q}_{3}\right)^{2}+M_{P}^{2}}-\left|U_{F S I}\right|+\left|V_{e f f}^{P}\right| \\
& T^{P}=\sqrt{\boldsymbol{k}^{2}+M_{P}^{2}} \quad \epsilon^{P}=\epsilon_{S M}^{P}-\left\langle T^{P}\right\rangle \\
& \left.v+\left[\left(M_{P}+T^{P}\right)-x^{S M}\right)\right]=\sqrt{\left(\mathbf{k}+\boldsymbol{q}_{3}\right)^{2}+M_{P}^{2}} \\
& x^{S M}=\epsilon_{S M}^{\prime P}-\left|U_{F S I}\right|+\left|V_{e f f}^{P}\right| \\
& Q^{2}=4\left(E_{0}+\left|V_{e f f}\right|\right)\left(E^{\prime}+\left|V_{e f f}\right|\right) \sin ^{2}(\theta / 2) \\
& E^{\prime}=E_{0}-v, \quad E_{f}^{P}=v-\epsilon^{P}, \quad \boldsymbol{q}_{3}^{2}=Q^{2}+v^{2},
\end{aligned}
$$

and $U_{F S I}=U_{F S I}\left(\left(\boldsymbol{q}_{3}+\boldsymbol{k}\right)^{2}\right)$.

\subsection{Extraction of $U_{F S I}$ from in inclusive e-A QE data}

We define $k_{z}$ as the component of $\mathrm{k}$ along the direction of the of the 3-momentum transfer $\boldsymbol{q}_{3}$.

$$
\begin{aligned}
v+\left(M_{P}-\epsilon^{P}\right)= & \sqrt{\left\langle\boldsymbol{k}^{2}\left(k_{z}\right)\right\rangle+2 k_{z} \boldsymbol{q}_{3}+\boldsymbol{q}_{\mathbf{3}}^{\mathbf{2}}+M_{P}^{2}} \\
& -\left|U_{F S I}\left(\left\langle\boldsymbol{k}^{2}\left(k_{z}\right)\right\rangle+2 k_{z} \boldsymbol{q}_{3}+\boldsymbol{q}_{\mathbf{3}}^{\mathbf{2}}\right)\right|+\left|V_{e f f}^{P}\right| \\
\boldsymbol{q}_{\mathbf{3}}^{\mathbf{2}}= & Q^{2}+v^{2} \\
Q^{2}= & 4\left(E_{0}+\mid V_{e f f}\right)\left(E_{0}-v+\left|V_{e f f}\right|\right) \sin ^{2} \frac{\theta}{2}
\end{aligned}
$$

where in the calculation of $\boldsymbol{q}_{3}^{2}$ we have applied Coulomb corrections to the initial and final electron energies as described in Appendix A.

In the peak region of the $\mathrm{QE}$ distribution $k_{z} \approx 0$. Therefore, from the location of the peak in $v$ we extract $U_{F S I}\left(\left(\boldsymbol{q}_{3}+\boldsymbol{k}\right)^{2}\right)_{\text {peak }}$ for

$$
\begin{aligned}
\left(\boldsymbol{q}_{3}+\boldsymbol{k}\right)_{\text {peak }}^{2} & \approx\left\langle\boldsymbol{k}^{2}\left(k_{z}=0\right)\right\rangle+\boldsymbol{q}_{\mathbf{3}}^{\mathbf{2}} \\
& =\frac{1}{2} k_{F}^{2}+\boldsymbol{q}_{\mathbf{3}}^{\mathbf{2}} \\
& \approx 0.02 \mathrm{GeV} V^{2}+\boldsymbol{q}_{\mathbf{3}}^{\mathbf{2}} \quad\left(\text { for } K_{F}=0.2\right)
\end{aligned}
$$

where we have used Eq. 33 for the Fermi gas distribution. If simplicity is needed then $\left(\boldsymbol{q}_{3}+\boldsymbol{k}\right)^{2} \approx \boldsymbol{q}_{3}^{2}$ is a good approximation. 

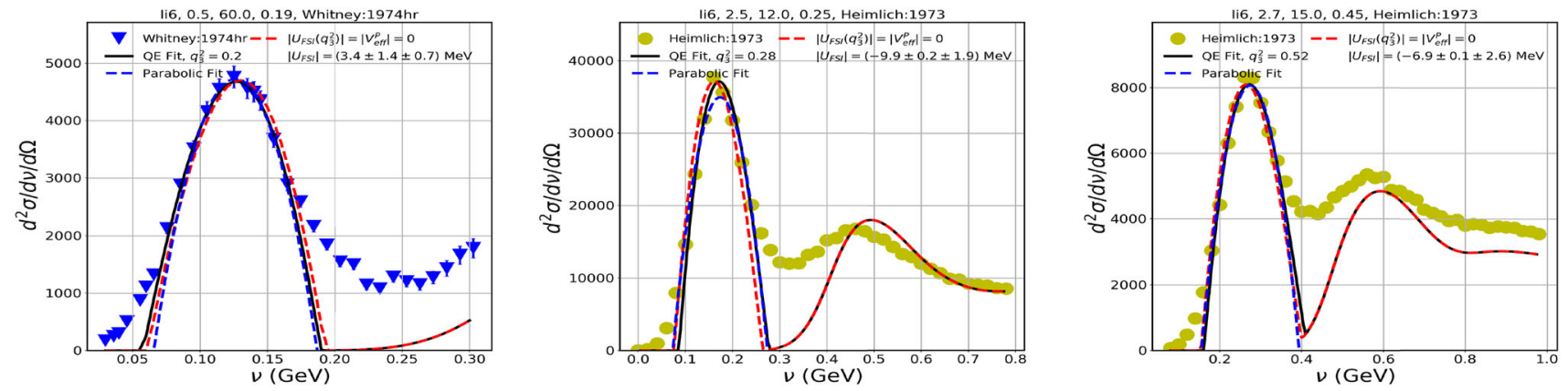

Fig. 8 Examples of fits for three out of four ${ }_{3}^{6} \mathrm{Li}\left(k_{F}^{P}=0.169 \mathrm{GeV}\right)$ QE differential cross sections. The solid blue curve is the RFG fit with the best value of $U_{F S I}$. The black dashed curve is the simple parabolic fit used to estimate the systematic error. The red dashed curve is the
RFG model with $U_{F S I}=V_{e f f}=0$. Above each panel we show: The element, $E_{0}$ in $\mathrm{GeV}, \theta$ in degrees, $Q^{2}$ in $\mathrm{GeV}^{2}$, First Author, and year of publication
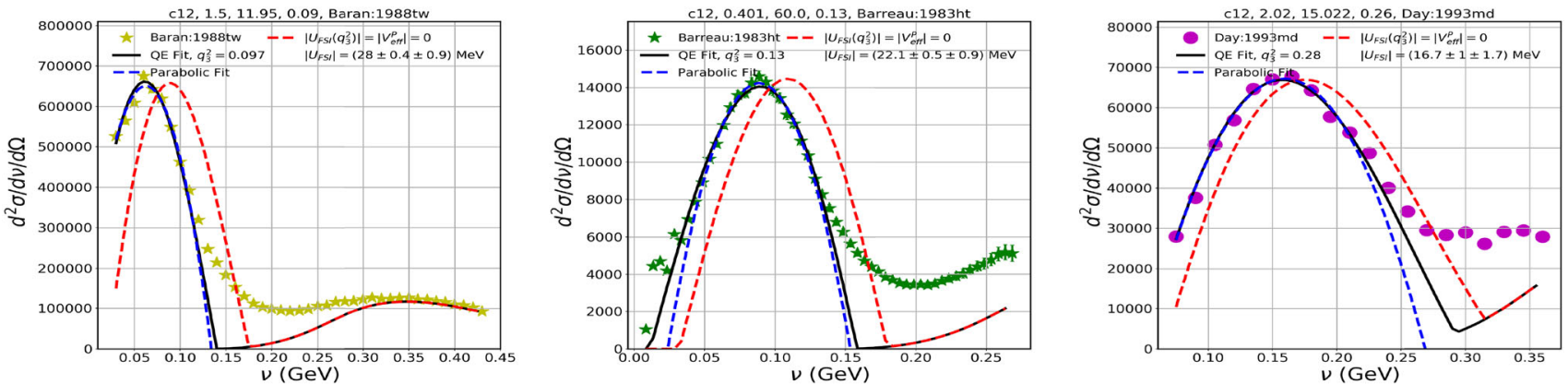

Fig. 9 Same as Fig. 8 for three out of $33{ }_{6}^{12} \mathrm{C}\left(k_{F}^{P}=0.221 \mathrm{GeV}\right) \mathrm{QE}$ differential cross sections

We fit a large number of electron scattering QE differential cross sections for various nuclei and extract the values of $U_{F S I}\left(\left(\boldsymbol{q}_{3}+\boldsymbol{k}\right)_{\text {peak }}^{2}\right)$. The data samples include: four ${ }_{3}^{6} \mathrm{Li}$ spectra, $33{ }_{6}^{12} \mathrm{C}$ spectra, five ${ }_{8}^{16} \mathrm{O}$ spectra, seven ${ }_{18}^{27} \mathrm{Al}$ spectra, $29{ }_{20}^{40} \mathrm{Ca}$ spectra, two ${ }_{18}^{40} \mathrm{Ar}$ spectra, $30{ }_{26}^{56} \mathrm{Fe}$ spectra, $23{ }_{82}^{208} \mathrm{~Pb}$ spectra and one ${ }_{79}^{197} \mathrm{Au}$ ) spectra. Most (but not all) of the QE differential cross sections given in references [55-78]) are available on the QE electron scattering archive [53,54]. Figures $8,9,10,11,12,13,14,15$ show examples of these fits to QE differential cross sections for all these elements. The solid blue curve is the RFG fit with the best value of $U_{F S I}$. The black dashed curve is a simple parabolic fit used to estimate the systematic error. The red dashed curve is the RFG model with $U_{F S I}=V_{\text {eff }}=0$.

The extracted values of $U_{F S I}$ versus $\left(\boldsymbol{q}_{3}+\boldsymbol{k}\right)^{2}$ for Lithium, Carbon+Oxygen, Aluminum, Calcium +Argon, iron, and Lead+Gold are shown in Figs. 16, 17, 18, 19, 20, and 21, respectively. Here $\left(\boldsymbol{q}_{3}+\boldsymbol{k}\right)^{2}$ is evaluated at the peak of the QE distribution. We fit the extracted values of $U_{F S I}\left(\left(\boldsymbol{q}_{3}+\boldsymbol{k}\right)^{2}\right)$ versus $\left(\boldsymbol{q}_{3}+\boldsymbol{k}\right)^{2}$ for $\left(\boldsymbol{q}_{3}+\boldsymbol{k}\right)^{2}>0.1 \mathrm{GeV}^{2}$ to a linear function. The intercepts at $\left(\boldsymbol{q}_{3}+\boldsymbol{k}\right)^{2}=0$ and the slopes of $U_{F S I}\left(\left(\boldsymbol{q}_{3}+\boldsymbol{k}\right)^{2}\right)$ are given in Table 9 for various nuclei.

For the Relativistic Fermi Gas (RFG) the probability distribution $P_{r f g}\left(k_{z}\right)$ and the average $\left\langle\boldsymbol{k}^{2}\left(k_{z}\right)\right\rangle_{r f g}$ are given in
Appendix B. We compare the e-A QE cross sections versus $v$ to the RFG model for QE scattering. We account for the nucleon $Q^{2}$ dependent form factors and for Pauli suppression (discussed in Appendix B.2) at low $\boldsymbol{q}_{3}^{2}$.

We only fit to the data in the top $1 / 3$ of the QE distribution to extract the best value of $U_{F S I}$ for $\left(\boldsymbol{q}_{3}+\boldsymbol{k}\right)^{2}$ at the peak. In the fit we let the normalization of the QE peak float to agree with data. For the estimate of the systematic error we also fit the QE differential cross section versus $v$ near the peak region to a simple parabola and extract the value of $v_{\text {peak }}^{\text {parabola }}$. We use the difference between $v_{\text {peak }}^{\text {parabola }}$ and $v_{\text {peak }}^{r f g}$ as a systematic error in our extraction of $U_{F S I}\left(\left(\boldsymbol{q}_{3}+\boldsymbol{k}\right)^{2}\right)$.

6.3 The $\Delta(1232)$ resonance shown in Figs. 8, 9, 10, 11, 12, 13,14 and 15

A simple calculation of the cross section for the production of $\Delta(1232)$ resonance is shown in Figs. 8, 9, 10, 11, 12, 13,14 and 15 . The calculation uses Jlab fits to the structure functions in the resonance region for protons and neutrons. These structure functions were extracted from hydrogen and deuterium data.

The proton and neutron structure functions in the resonance region were used as input to a simple Fermi Gas 

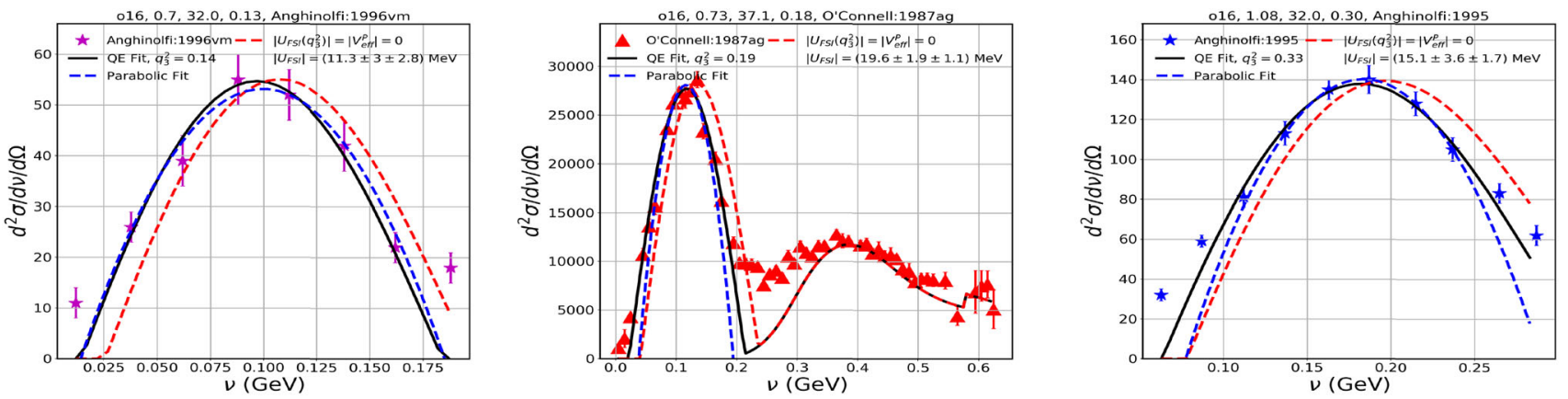

Fig. 10 Same as Fig. 8 for three out of four ${ }_{8}^{16} \mathrm{O}\left(k_{F}^{P}=0.225 \mathrm{GeV}\right)$ QE differential cross sections
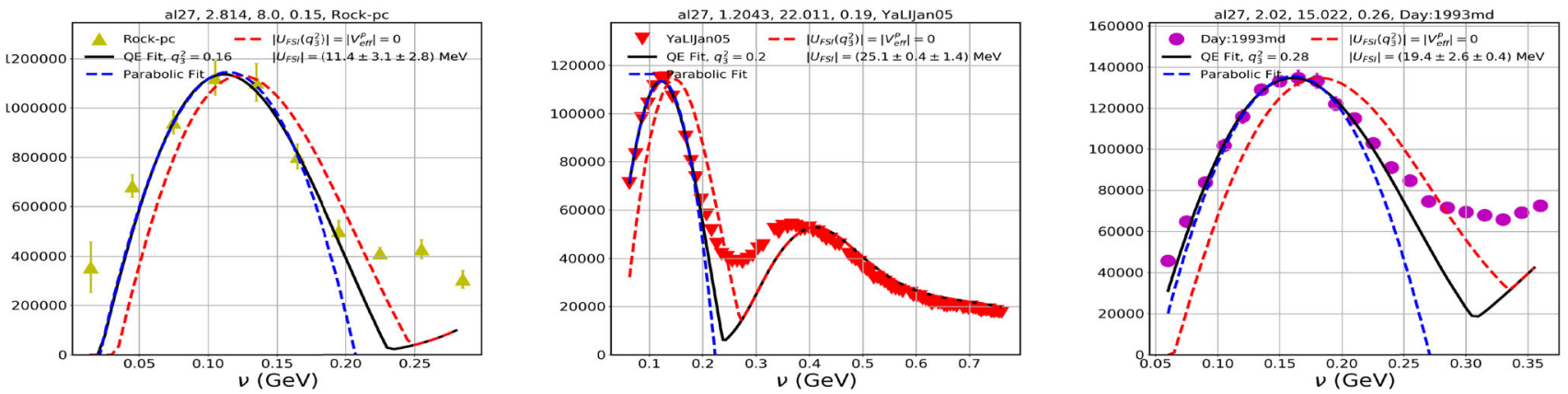

Fig. 11 Same as Fig. 8 for three out of seven ${ }_{13}^{27} \mathrm{Al}\left(k_{F}^{P}=0.238 \mathrm{GeV}\right) \mathrm{QE}$ differential cross sections
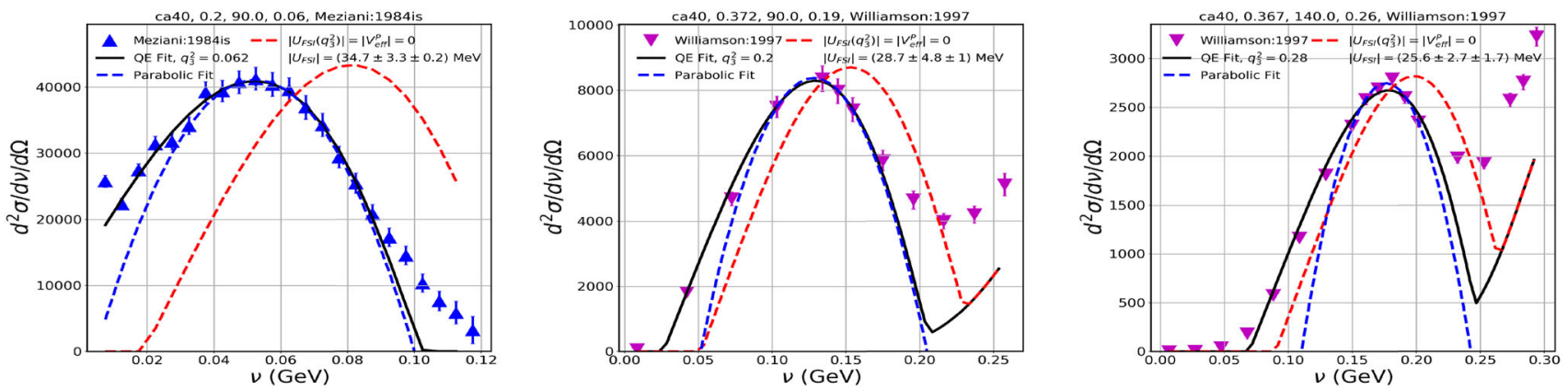

Fig. 12 Same as Fig. 8 for three out of $29{ }_{20}^{40} \mathrm{Ca}\left(k_{F}^{P}=0.251 \mathrm{GeV}\right) \mathrm{QE}$ differential cross sections
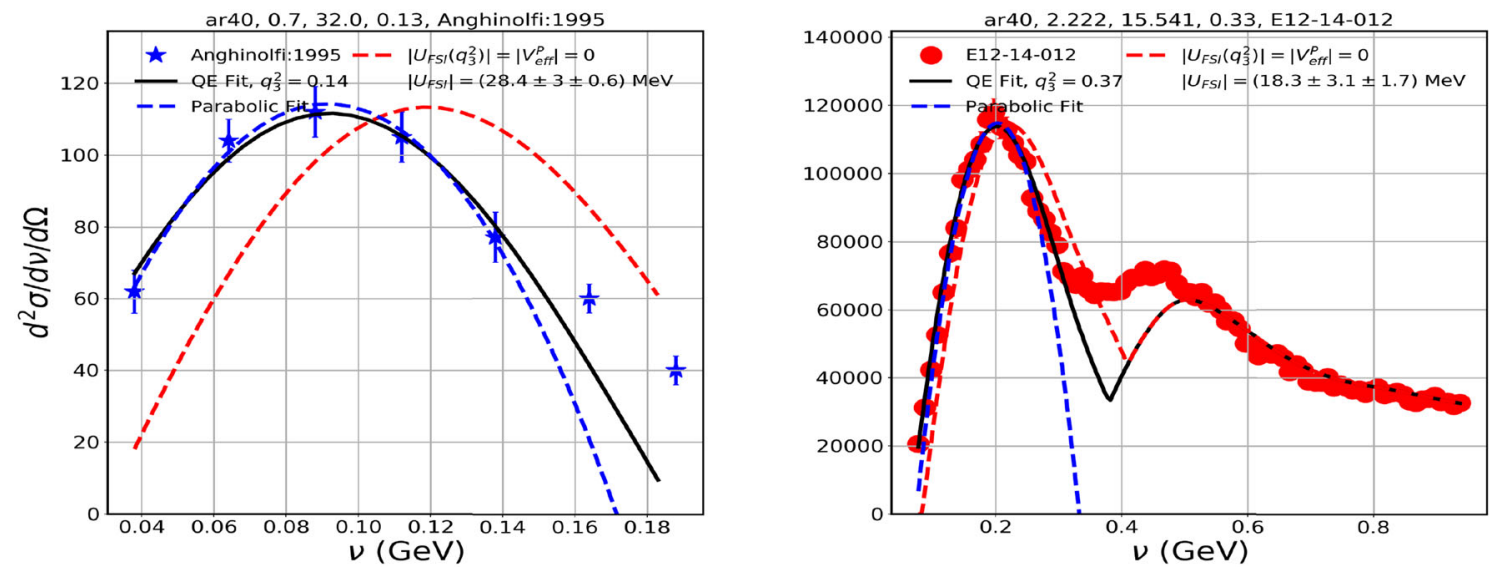

Fig. 13 Same as Fig. 8 for two ${ }_{18}^{40} \operatorname{Ar}\left(k_{F}^{P}=0.251 \mathrm{GeV}\right) \mathrm{QE}$ differential cross sections 

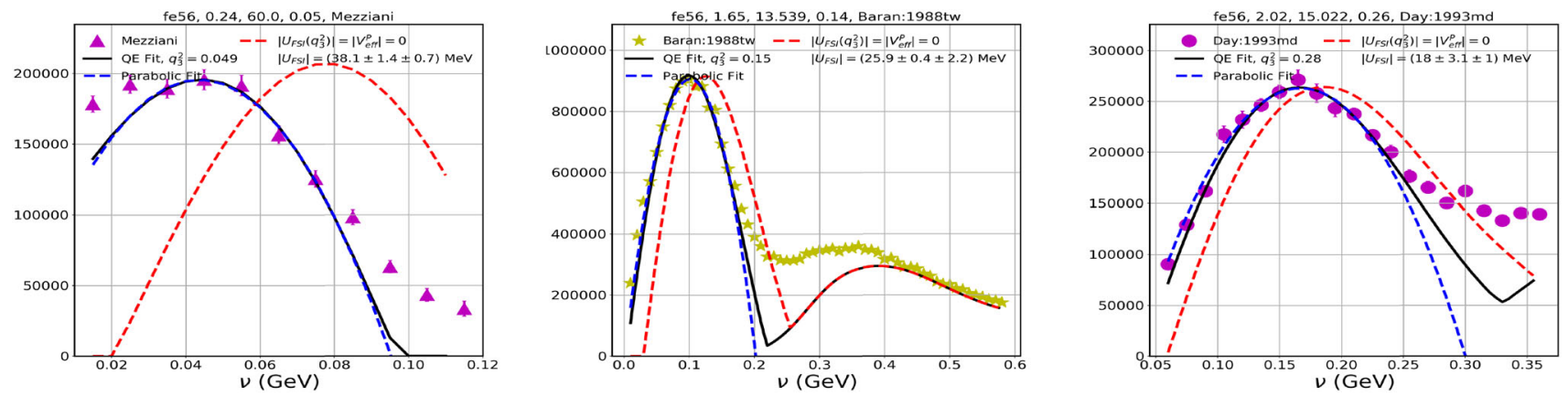

Fig. 14 Same as Fig. 8 for three of $30{ }_{20}^{56} \mathrm{Fe}\left(k_{F}^{P}=0.254 \mathrm{GeV}\right)$ QE differential cross sections
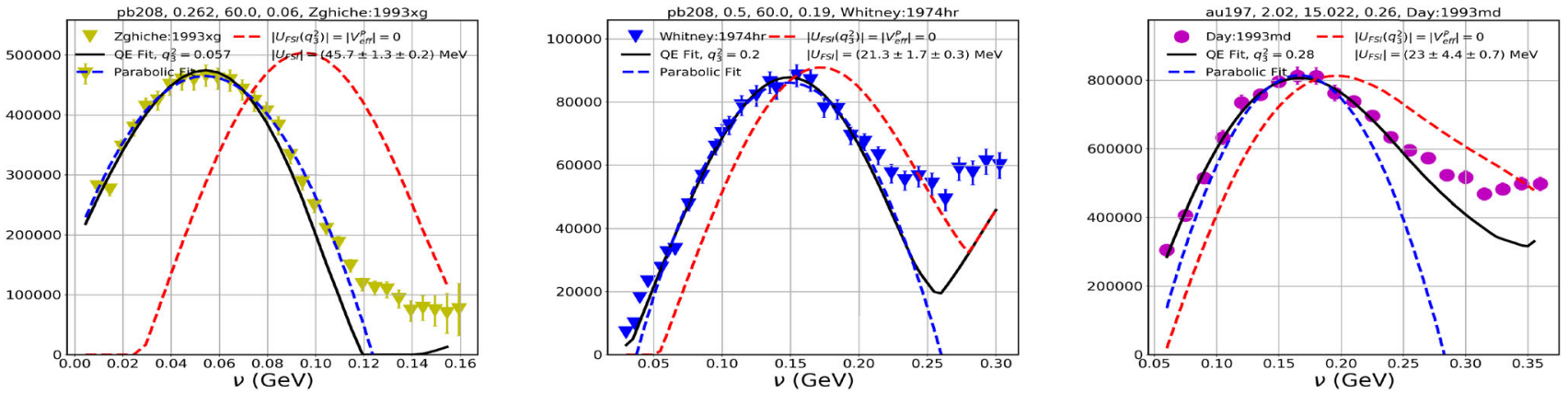

Fig. 15 Same as Fig. 8 for two of $22{ }_{82}^{208} \mathrm{~Pb}\left(k_{F}^{P}=0.275 \mathrm{GeV}\right)$ and one ${ }_{79}^{197} \mathrm{Au}\left(k_{F}^{P}=0.275 \mathrm{GeV}\right) \mathrm{QE}$ differential cross sections

Fig. 16 Extracted values of $U_{F S I}$ versus $\left(\boldsymbol{q}_{3}+\boldsymbol{k}\right)^{2}$ for four Lithium $\left({ }_{3}^{6} \mathrm{Li}\right)$ spectra

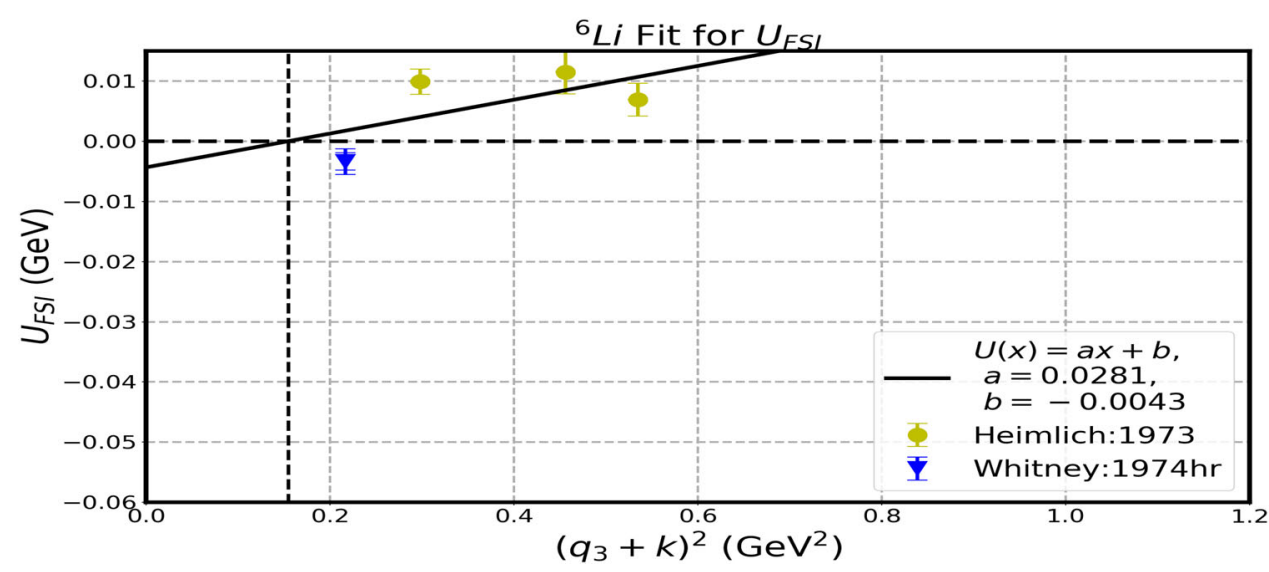

smearing model. In the calculation, $U_{F S I}$ for the $\Delta(1232)$ resonance is assumed to be the same as $U_{F S I}$ for QE scattering.

The curves shown Figs. 8, 9, 10, 11, 12, 13, 14 and 15 do not include the contributions of $2 \mathrm{p} 2 \mathrm{~h}$ final states from meson exchange currents (MEC) and isobar excitation. These $2 \mathrm{p} 2 \mathrm{~h}$ contributions yield additional cross section in the region between the QE peak and the $\Delta(1232)$ resonance. The $2 \mathrm{p} 2 \mathrm{~h}$ contributions are primarily transverse and therefore are more significant for electron scattering at larger angles than at small angles (as observed in the figures). The investigations of MEC (which is model dependent) and the values of $U_{F S I}$ for a $\Delta(1232)$ resonance in the final state are the subject of a future investigation.

\section{Implementation for neutrino experiments}

For QE scattering of neutrinos (antineutrinos) on bound neutrons (protons) the final state nucleon is a proton (neutron). The following equations should be used in neutrino/antineutrino MC generators:

For neutrino QE scattering on bound neutrons:

$v+\left(M_{N}-\epsilon^{N}\right)=\sqrt{\left(\mathbf{k}+\boldsymbol{q}_{\mathbf{3}}\right)^{2}+M_{P}^{2}}-\left|U_{F S I}\right|+\left|V_{e f f}^{P}\right|$

where $\left|V_{e f f}^{P}\right|=\left|V_{e f f}\right|$. 


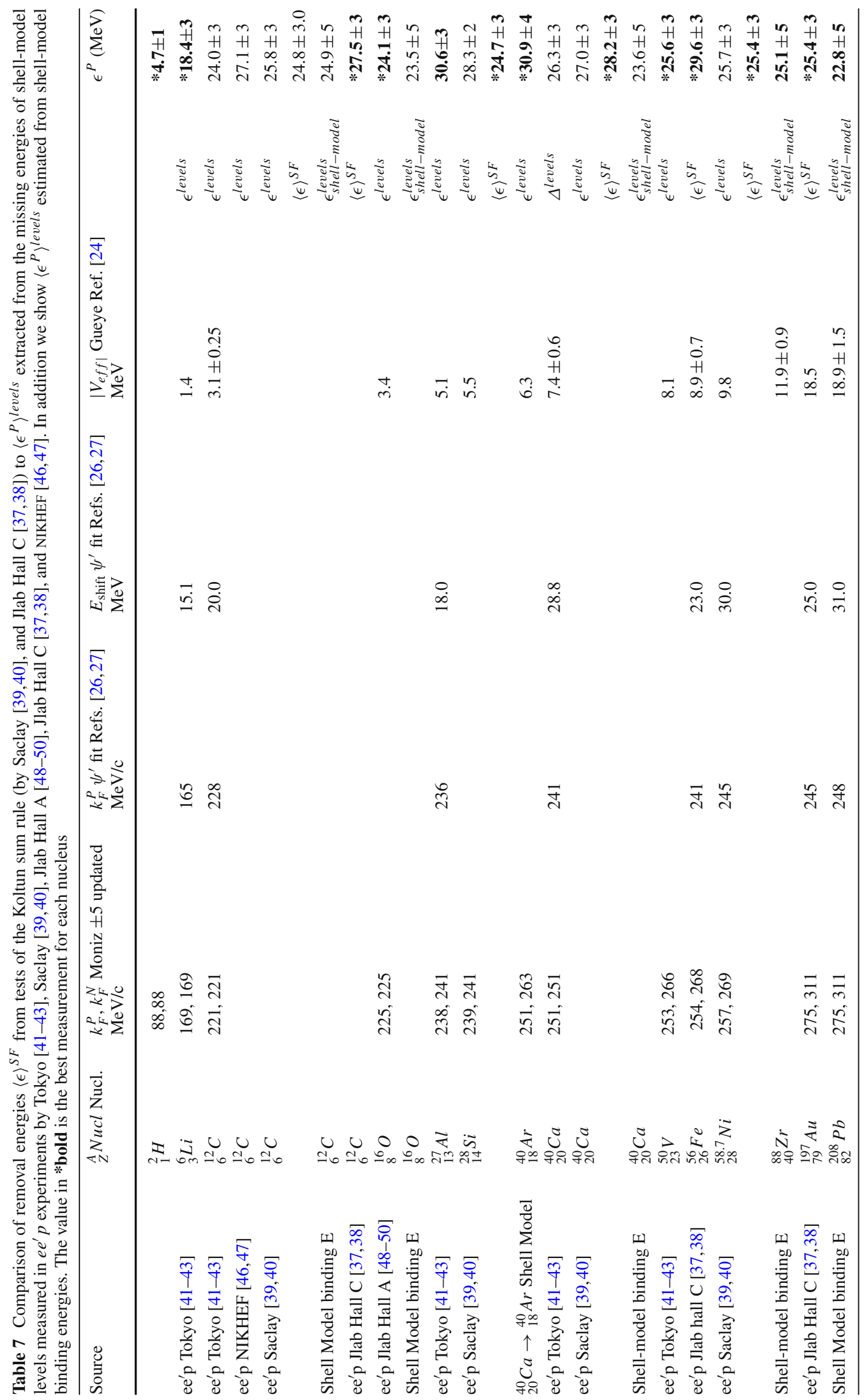




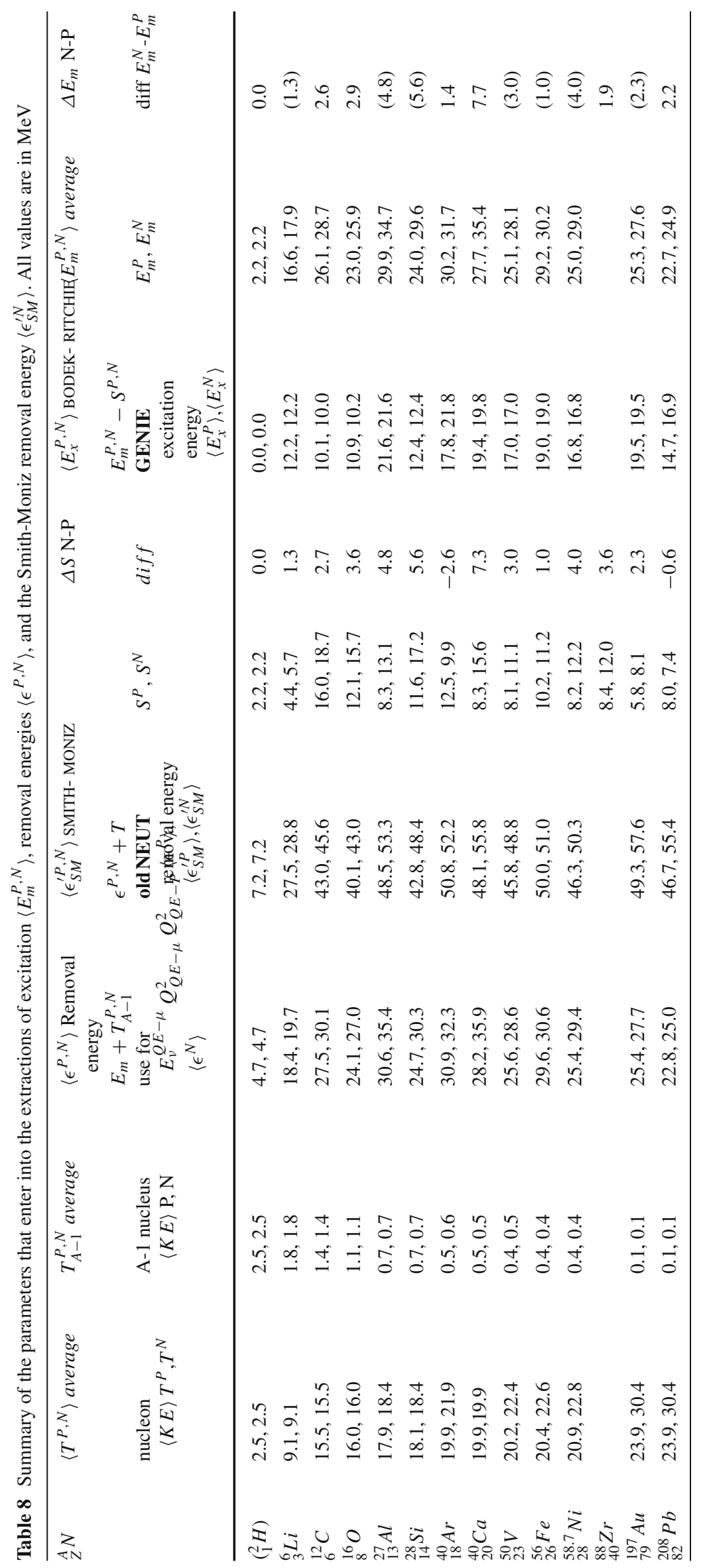


Fig. 17 Extracted values of $U_{F S I}$ versus $\left(\boldsymbol{q}_{3}+\boldsymbol{k}\right)^{2}$ for 33 Carbon $\left({ }_{6}^{12} \mathrm{C}\right)$ and 8 Oxygen $\left({ }_{8}^{16} \mathrm{O}\right)$ spectra

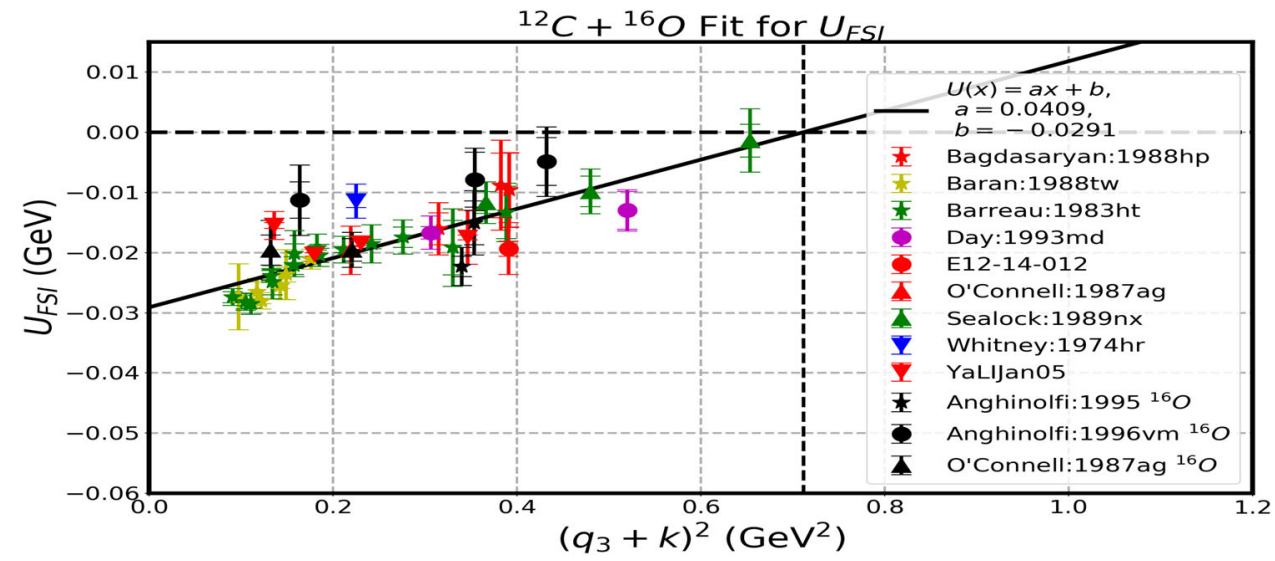

For antineutrino QE scattering on bound protons:

$v+\left(M_{P}-\epsilon^{P}\right)=\sqrt{\left(\mathbf{k}+\mathbf{q}_{\mathbf{3}}\right)^{\mathbf{2}}+M_{N}^{2}}-\left|U_{F S I}\right|$

Where

$\epsilon^{N, P}=S^{N, P}+\left\langle E_{x}^{N, P}\right\rangle+\frac{\left\langle k^{2}\right\rangle}{2 M_{A-1}^{*}}$
Rearranging, we have

$v+\left(M_{N, P}-x^{\nu, \bar{v}}\right)=\sqrt{\left(k+q_{3}\right)^{2}+M_{P, N}^{2}}$

where for neutrinos and antineutrinos we have:

$x^{\nu}\left(\left(\boldsymbol{q}_{3}+\boldsymbol{k}\right)^{2}\right)=\epsilon^{N}-\left|U_{F S I}\right|+\left|V_{e f f}^{P}\right|$
$x^{\bar{v}}\left(\left(\boldsymbol{q}_{3}+\boldsymbol{k}\right)^{2}\right)=\epsilon^{P}-\left|U_{F S I}\right|$
Fig. 18 Extracted values of $U_{F S I}$ versus $\left(\boldsymbol{q}_{3}+\boldsymbol{k}\right)^{2}$ for 8 Aluminum $\left({ }_{13}^{27} \mathrm{Al}\right)$ spectra

Fig. 19 Extracted values of $U_{F S I}$ versus $\left(\boldsymbol{q}_{3}+\boldsymbol{k}\right)^{2}$ for 29 Calcium $\left({ }_{20}^{40} \mathrm{Ca}\right)$ and 2 $\operatorname{Argon}\left({ }_{18}^{40} \mathrm{Ar}\right)$ spectra
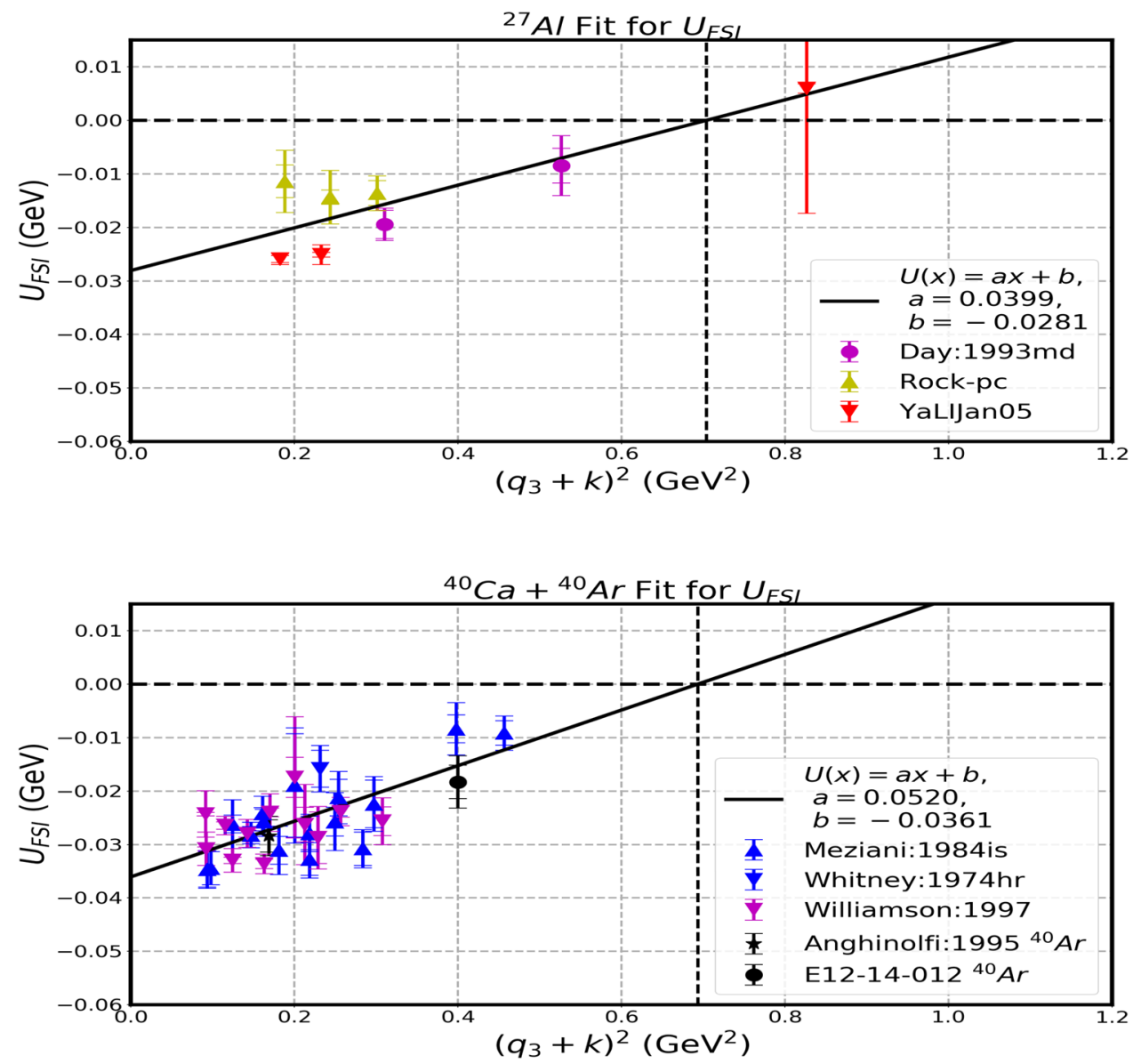
Fig. 20 Extracted values of $U_{F S I}$ versus $\left(\boldsymbol{q}_{3}+\boldsymbol{k}\right)^{2}$ for 30 Iron $\left({ }_{26}^{56} \mathrm{Fe}\right)$ spectra

Fig. 21 Extracted values of $U_{F S I}$ versus $\left(\boldsymbol{q}_{3}+\boldsymbol{k}\right)^{2}$ for 22 Lead $\left({ }_{82}^{208} \mathrm{~Pb}\right)$ and one Gold $\left({ }_{79}^{197} \mathrm{Au}\right)$ spectra
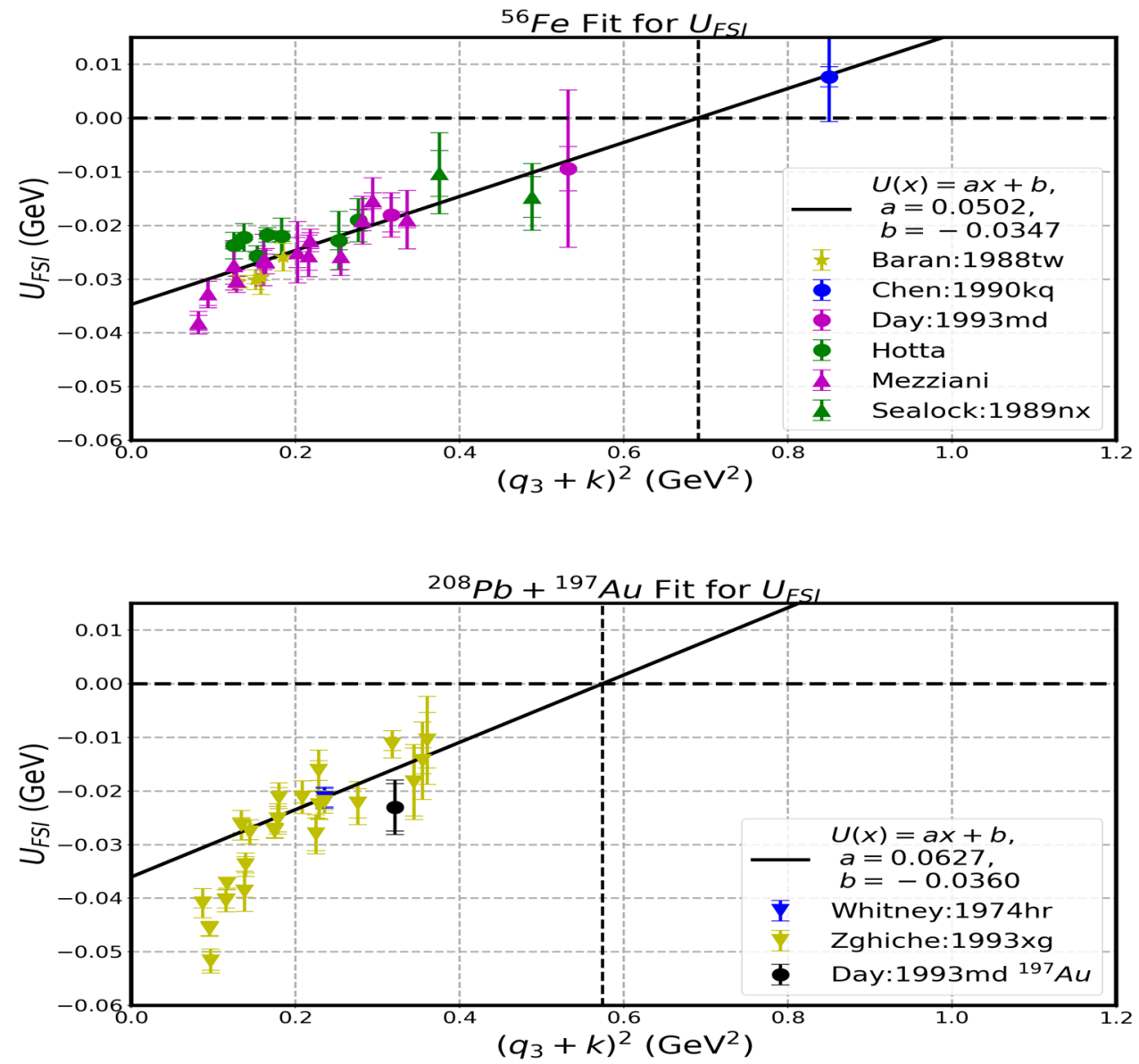

\section{Neutrino scattering on neutron}

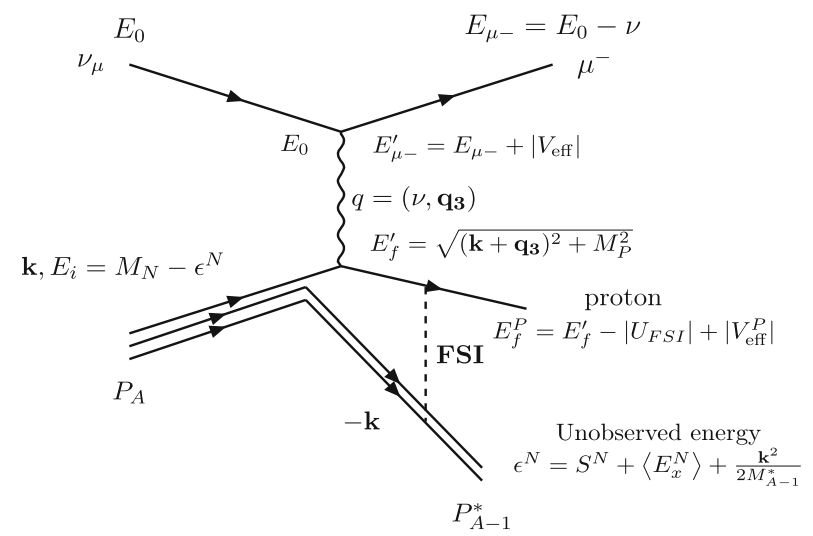

Fig. 22 1p1h process: Neutrino (left) and antineutrino (right) QE scattering from an off-shell bound nucleon of momentum $\boldsymbol{p}_{\boldsymbol{i}}=\boldsymbol{k}$ in a nucleus of mass A. The off-shell energy of the interacting nucleon is $E_{i}^{N, P}=M_{N, P}-\epsilon^{N, P}$, where $\epsilon^{N, P}=S^{N, P}+\left\langle E_{x}^{N, P}\right\rangle+\frac{\boldsymbol{k}^{2}}{2 M_{A-1}^{*}}$. We model the effect of FSI (strong and EM interactions) by setting the energy of the final state proton $E_{f}^{P}=\sqrt{\left(\boldsymbol{k}+\boldsymbol{q}_{3}\right)^{2}+M_{P}^{2}}-\left|U_{F S I}\right|+$ $\left|V_{e f f}^{P}\right|$ for neutrino QE scattering on bound neutrons, and the energy of
Anti-Neutrino scattering on proton

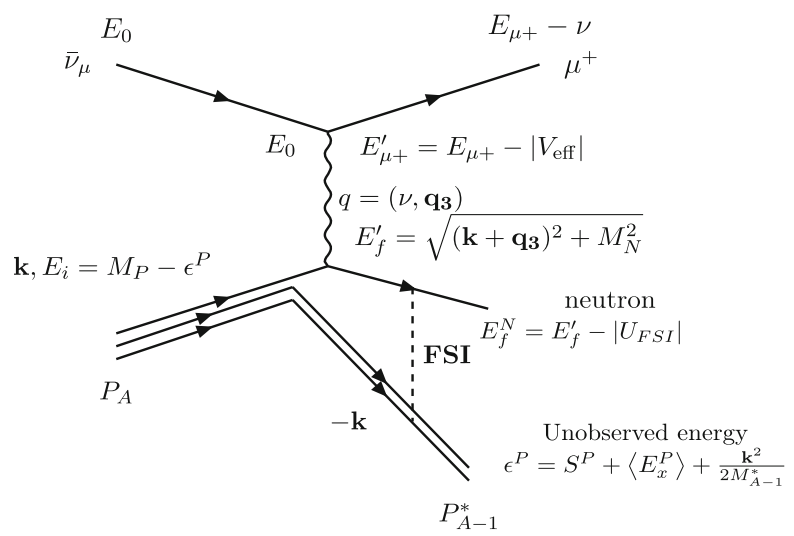

the final state neutron $E_{f}^{N}=\sqrt{\left(\boldsymbol{k}+\boldsymbol{q}_{\mathbf{3}}\right)^{2}+M_{P}^{2}}-\left|U_{F S I}\right|$ for antineutrinos scattering on bound protons. Here $U_{F S I}=U_{F S I}\left(\left(\boldsymbol{q}_{3}+\boldsymbol{k}\right)^{2}\right)$. For neutrino QE scattering on bound neutrons $\left|V_{e f f}^{P}\right|=\left|V_{\text {eff }}\right|$, and the effective $\mu$ - energy at the vertex is $E_{\mu-}^{\prime}=E_{\mu-}+V_{e f f}$. For antineutrino QE scattering on bound protons $\left|V_{e f f}^{N}\right|=0$ for the final state neutron and the effective $\bar{\mu}$ energy at the vertex is $E_{\bar{\mu}}^{\prime}=E_{\bar{\mu}}-V_{\text {eff }}$ 
Table 9 The intercepts $(\mathrm{GeV})$ at $\boldsymbol{q}_{\mathbf{3}}^{\mathbf{2}}=0$ and slopes $\left(\mathrm{GeV} / \mathrm{GeV}^{2}\right)$ of fits to $U_{F S I}$ versus $\left(\boldsymbol{q}_{3}+\boldsymbol{k}\right)^{2}$. The overall systematic errors on $U_{F S I}$ are estimated at $\pm 0.005 \mathrm{GeV}$

\begin{tabular}{llll}
\hline$Z^{\mathbf{A}} \mathbf{N u c l}$ & $\left|V_{\text {eff }}\right|$ & $\begin{array}{l}U_{F S I} \\
\text { intercept } \\
\left(\boldsymbol{q}_{3}+\boldsymbol{k}\right)^{2}=0 .\end{array}$ & $\begin{array}{l}U_{F S I} \\
\text { slope vs } \\
\left(\boldsymbol{q}_{3}+\boldsymbol{k}\right)^{2}\end{array}$ \\
\hline $\mathbf{3}^{\mathbf{6}} \mathbf{L i}$ & 0.0014 & -0.0043 & 0.0281 \\
$\mathbf{6}^{\mathbf{1 2}} \mathbf{C} / \mathbf{8}^{\mathbf{1 6}} \mathbf{O}$ & 0.0031 & -0.0291 & 0.0409 \\
$\mathbf{1 3}^{\mathbf{2 7}} \mathbf{A l}$ & 0.0051 & -0.0281 & 0.0399 \\
$\mathbf{2 0}^{\mathbf{4 0}} \mathbf{C a} /{ }_{\mathbf{1 0}}^{\mathbf{4 0}} \mathbf{A r}$ & $0.0074 / 0.0063$ & -0.0361 & 0.0520 \\
$\mathbf{2 6}^{\mathbf{5 6}} \mathbf{F e}$ & 0.0089 & -0.0347 & 0.0502 \\
$\mathbf{8 2}^{\mathbf{2 0 8}} \mathbf{P b} / \mathbf{7 9}^{\mathbf{1 9 7}} \mathbf{A u}$ & $0.0189 / 0.0185$ & -0.0360 & 0.0627 \\
\hline
\end{tabular}

and

$$
\begin{aligned}
Q^{2} & =-m_{\mu, \bar{\mu}}^{2}+2 E_{v, \bar{v}}\left(E_{\mu, \bar{\mu}}^{\prime}-\sqrt{\left(E_{\mu, \bar{\mu}}^{\prime}\right)^{2}-m_{\mu, \bar{\mu}}^{2}} \cos \theta_{\mu, \bar{\mu}}\right) \\
E_{\mu, \bar{\mu}} & =E_{v, \bar{v}}-v ; \quad \boldsymbol{q}_{3}^{2}=Q^{2}+v^{2} \\
E_{\mu}^{\prime} & =E_{\mu}+\left|V_{e f f}\right| ; \quad E_{\bar{\mu}}^{\prime}=E_{\bar{\mu}}-\left|V_{e f f}\right| \\
E_{f}^{P, N} & =v-\epsilon^{N, P}
\end{aligned}
$$

For both neutrinos and antineutrinos $\epsilon^{P, N}$ is the unobserved removal energy (Fig. 22).

In neutrino experiments in which both the final state lepton and final state proton (or neutron) are measured (e.g. NOVA, MINERVA, DUNE) the neutrino energy can be calculated as follows:

$$
\begin{aligned}
E_{v, \bar{v}} & =E_{\mu, \bar{\mu}}+E_{f}^{P, N}+\epsilon^{N, P} \\
\epsilon^{N, P} & =S^{N, P}+\left\langle E_{x}^{N, P}\right\rangle+T_{A-1}^{N, P}
\end{aligned}
$$

$T_{A-1}^{N, P}=\frac{\left\langle\left(k^{N, P}\right)^{2}\right\rangle}{2 M_{A-1}}=\frac{3}{5} \frac{\left(k_{F}^{N, P}\right)^{2}}{2 M_{A-1}}$

\section{Corrections to GENIE version 2}

The generation of events in GENIE 2 as currently done is equivalent to using Eqs. 19 and 20, but with $V_{\text {eff }}=0$, $U_{F S I}=0$, and $\left\langle E_{x}^{P, N}\right\rangle=0$. In addition, an amount $\Delta_{\mathrm{GENIE}}^{\text {nucleon }}$ (25 MeV for ${ }_{6}^{12} C$ ) is subtracted from the energy of the final state nucleon (or quark for inelastic events) to account for "binding energy" in GENIE 2. For neutrino QE scattering on bound neutrons events are generated in GENIE 2 using the following equations:

$$
\begin{aligned}
& v_{\mathrm{GENIE}}^{\nu, \bar{v}}+\left(M_{N, P}-x_{\mathrm{GENIE}}^{\nu, \bar{v}}\right)=\sqrt{\left(\mathbf{k}+\boldsymbol{q}_{\mathbf{3}}\right)^{\mathbf{2}}+M_{P, N}^{2}} \\
& \epsilon_{\mathrm{GENIE}}^{\nu, \bar{v}}=x_{\mathrm{GENIE}}^{\nu, \bar{v}} ; \quad x_{\mathrm{GENIE}}^{\nu, \bar{v}}=S^{N, P}+\frac{\left\langle k^{2}\right\rangle}{2 M_{A-1}}
\end{aligned}
$$

Where $\epsilon_{\mathrm{GENIE}}^{\nu, \bar{v}}$ is the removal energy for neutrino (antineutrino) assumed in GENIE. Therefore the difference between the correct muon energy and the muon energy generated by GENIE is approximately equal to $\Delta x_{\mathrm{GENIE}}^{v}=x^{v}-x_{\mathrm{GENIE}}$.

$$
\begin{aligned}
\Delta x_{\mathrm{GENIE}}^{v} & =\left\langle E_{x}^{N}\right\rangle-\left|U_{F S I}\right|+\left|V_{e f f}^{P}\right| \\
\Delta x_{\mathrm{GENIE}}^{\bar{v}} & =\left\langle E_{x}^{P}\right\rangle-\left|U_{F S I}\right| \\
\Delta \epsilon_{\mathrm{GENIE}}^{\nu, \bar{v}} & =\left\langle E_{x}^{N, P}\right\rangle \\
\Delta \nu_{\mathrm{GENIE}}^{v, \bar{v}} & =v^{\nu, \bar{v}}-v_{\mathrm{GENIE}}^{\nu, \bar{v}} \\
& \approx \Delta x_{\mathrm{GENIE}}^{\nu, \bar{v}} \\
\Delta\left(E_{\mu}\right)_{\mathrm{GENIE}} & =E_{\mu}-\left(E_{\mu}\right)_{\mathrm{GENIE}} \\
& =-\Delta v_{\mathrm{GENIE}}^{v, \bar{v}}
\end{aligned}
$$

Table 10 Estimates of the difference between the correctly simulated muon, final state nucleon and removal (unobserved) energies and those generated by GENIE 2 for QE events in carbon

Table 11 Difference between the correctly simulated muon, nucleon and removal (unobserved) energies and those generated by GENIE 2 for QE events in Oxygen

\begin{tabular}{lllll}
\hline Carbon MC & $\boldsymbol{q}_{3}^{2} \mathrm{GeV}^{2}$ & $\Delta E_{\mu+, \mu-} \mathrm{MeV}$ & $\Delta E_{f}^{P, N} \mathrm{MeV}$ & $\Delta \epsilon^{P, N} \mathrm{MeV}$ \\
\hline$U_{F S I}=20.0 \mathrm{MeV}$ & 0.2 & & & \\
GENIE $v$ & +6.9 & +8.1 & +10.0 \\
GENIE $\bar{v}$ & & +9.9 & +5.0 & +10.1 \\
$U_{F S I}=0.0 \mathrm{MeV}$ & 0.8 & & & +10.0 \\
GENIE $v$ & & -13.1 & +28.1 & +10.1 \\
GENIE $\bar{v}$ & -10.1 & +25.0 & \\
\hline
\end{tabular}

\begin{tabular}{lllll}
\hline Oxygen MC & $q_{3}^{2} \mathrm{GeV}^{2}$ & $\Delta E_{\mu+, \mu-} \mathrm{MeV}$ & $\Delta E_{f}^{P, N} \mathrm{MeV}$ & $\Delta \epsilon^{P, N} \mathrm{MeV}$ \\
\hline$U_{F S I}=20.0 \mathrm{MeV}$ & 0.2 & & & \\
GENIE $v$ & +6.4 & +8.4 & +10.2 \\
GENIE $\bar{v}$ & & +9.1 & +5.0 & +10.9 \\
$U_{F S I}=0.0 \mathrm{MeV}$ & 0.8 & & & +10.2 \\
GENIE $v$ & & -13.6 & +28.4 & +10.9 \\
GENIE $\bar{v}$ & & -10.9 & +25.0 & \\
\hline
\end{tabular}




$$
\begin{aligned}
\Delta\left(E_{f}^{P, N}\right)_{\mathrm{GENIE}} & =E_{f}^{P, N}-\left(E_{f}^{P, N}\right)_{\mathrm{GENIE}} \\
& =\Delta v_{\mathrm{GENIE}}^{\nu, \bar{v}}-\left\langle E_{x}^{N, P}\right\rangle+\Delta_{\mathrm{GENIE}}^{\text {nucleon }}
\end{aligned}
$$

Where $\Delta_{\text {GENIE }}^{\text {nucleon }}=25 \mathrm{MeV}$ is used in GENIE.

As given in the Tables 7 and $8,\left\langle E_{x}^{P, N}\right\rangle$ for Carbon is equal to $10.1 \mathrm{MeV}$ and $10.0 \mathrm{MeV}$ for protons and neutrons, respectively and $\left|V_{e f f}\right|=3.1 \mathrm{MeV}$.

For Oxygen $\left\langle E_{x}^{P, N}\right\rangle$ is equal to $10.9 \mathrm{MeV}$ and 10.2 $\mathrm{MeV}$ for protons and neutrons, respectively, and $\left|V_{\text {eff }}\right|=$ 3.4 MeV.

For Argon $\left\langle E_{x}^{P, N}\right\rangle$ is equal to $17.8 \mathrm{MeV}$ and $21.8 \mathrm{MeV}$ for protons and neutrons, respectively, and $\left|V_{\text {eff }}\right|=6.3 \mathrm{MeV}$ (Table 9).

Tables 10 and 11 show the differences between the correctly simulated muon, final state nucleon and removal (unobserved) energies and those generated by GENIE 2 for QE events in carbon, and oxygen respectively. These differences are shown for the case of $\boldsymbol{q}_{3}^{2}=0.2 \mathrm{GeV}^{2}\left(\left|U_{F S I}\right|=\right.$ $20 \mathrm{MeV})$ and for $\boldsymbol{q}_{3}^{2}=0.8 \mathrm{GeV}^{2}\left(\left|U_{F S I}\right|=0\right)$.

\section{Conclusion}

We investigate the binding energy parameters that should be used in modeling electron and neutrino scattering from nucleons bound in a nucleus within the framework of the impulse approximation. We discuss the relation between binding energy, missing energy, removal energy $(\epsilon)$, spectral functions and shell model energy levels and extract updated removal energy parameters from ee' $\mathrm{p}$ spectral function data. We address the difference in parameters for scattering from bound protons and neutrons. We also use inclusive e-A data to extract an empirical parameter $U_{F S I}\left(\left(\boldsymbol{q}_{3}+\right.\right.$ $\boldsymbol{k})^{2}$ ) to account for the interaction of final state nucleons (FSI) with the optical potential of the nucleus. Similarly we use $V_{\text {eff }}$ to account for the Coulomb potential of the nucleus.

With three parameters $\epsilon, U_{F S I}\left(\left(\boldsymbol{q}_{3}+\boldsymbol{k}\right)^{2}\right)$ and $V_{\text {eff }}$ we can describe the energy of final state electrons for all available electron QE scattering data. The use of the updated parameters in neutrino Monte Carlo generators reduces the systematic uncertainty in the combined removal energy (with FSI corrections) from $\pm 20 \mathrm{MeV}$ to $\pm 5 \mathrm{MeV}$.

Data Availability Statement This manuscript has no associated data or the data will not be deposited. [Author's comment: For each nucleus there are four parameters: $\epsilon,\left|V_{e f f}\right|$ and two parameters for $U_{F S I}$. Neutrino experiments typically have one heavy target nucleus (e.g. Carbon, or Oxygen), for which the values can be taken directly from the tables.].

Open Access This article is distributed under the terms of the Creative Commons Attribution 4.0 International License (http://creativecomm ons.org/licenses/by/4.0/), which permits unrestricted use, distribution, and reproduction in any medium, provided you give appropriate credit to the original author(s) and the source, provide a link to the Creative
Commons license, and indicate if changes were made. Funded by SCOAP ${ }^{3}$.

\section{A Appendix: Coulomb corrections}

For targets with atomic number $\mathrm{Z}$ greater than one we should take into account the effect of the electric field of the nucleus on the incident and scattered electrons (and also on the final state proton in QE events). These corrections are called Coulomb corrections. For atomic weight $\mathrm{A}$ and atomic number $\mathrm{Z}$ the protons create an electrostatic potential $\mathrm{V}(\mathrm{r})$. In the effective momentum approximation (EMA), the effective potential for an incident electron is $V_{\text {eff }}$, which can be calculated as follows:

$$
\begin{aligned}
V(r) & =\frac{3 \alpha(Z)}{2 R}+\frac{r \alpha(Z)}{2 R^{2}} \\
R & =1.1 A^{1 / 3}+0.775 A^{-1 / 3} \\
V_{e f f} & =-0.8 V(r=0)=-0.8 \frac{3 \alpha(Z)}{2 R} .
\end{aligned}
$$

The values for $\left|V_{\text {eff }}\right|$ calculated from Eq. 29 agree (within errors) with values extracted from a comparison of the peak positions and cross sections of positron and electron QE scattering [24]. For our estimates of $\left|V_{e f f}\right|$ shown in Table 7 we use the experimental values for the nuclei that were measured in Ref. [24]. We use Eq. 29 to interpolate to other nuclei.

For electrons scattering on bound nucleons the effective incident energy is $E_{e f f}=E_{0}+\left|V_{e f f}\right|$, and the effective scattered energy is $E_{\text {eff }}^{\prime}=E^{\prime}+\left|V_{\text {eff }}\right|$. This implies that the effective square of the momentum transfer is increased. For positrons scattering on bound nucleons the effective incident energy is $E_{e f f}=E_{0}-\left|V_{e f f}\right|$, and the effective scattered energy is $E_{e f f}^{\prime}=E^{\prime}-\left|V_{e f f}\right|$. This implies that the effective square of the momentum transfer is decreased.

For electron QE scattering on bound protons $\left|V_{e f f}^{P}\right|=$ $\frac{Z-1}{Z}\left|V_{e f f}\right|$. For neutrino QE scattering on bound neutrons $\left|V_{e f f}^{P}\right|=\left|V_{e f f}\right|$. For neutrino QE scattering on bound protons $\left|V_{e f f}^{N}\right|=0$.

For completeness, though not relevant in this analysis, there is also a focusing factor $F_{f o c}=\left(\frac{E_{0}+\left|V_{\text {eff }}\right|}{E_{0}}\right)^{2}$ that enhances the cross section for electrons and reduces the cross section for positrons. The focussing factor cancels the $1 / E_{0}^{2}$ factor in the Mott cross section. Therefore, the Coulomb correction should only be applied to the structure functions $W_{1}$ and $W_{2}$.

\section{B Appendix: Relativistic Fermi Gas (RFG)}

For the Fermi gas model the momentum distribution is zero for $k>k_{F}$, and for $k<k_{F}$ it is given by 


$$
\begin{aligned}
|\phi(k)|^{2} & =\frac{1}{N_{r f g}} \quad N_{r f g}=\frac{4}{3} \pi K_{F}^{3} \\
P_{r f g}(k) d k & =|\phi(k)|^{2} 4 \pi \boldsymbol{k}^{2} d k=\frac{1}{N_{r f g}} 4 \pi \boldsymbol{k}^{2} d k,
\end{aligned}
$$

and $\left\langle\boldsymbol{k}^{2}\right\rangle=(3 / 5) k_{F}^{2}$.

B.1 Distributions and parameters of RFG versus $k_{z}$

Here we do the calculation in cylindrical coordinates

$$
\begin{aligned}
& \left(2 \pi \boldsymbol{k}^{2} d \cos \theta d k=\pi d k_{T}^{2} d k_{z}\right) \\
& k=\sqrt{k_{T}^{2}+k_{z}^{2}} .
\end{aligned}
$$

the probability distribution of the $\mathrm{Z}$ component of the momentum $k_{z} P\left(k_{z}\right) d k_{z}$, and average square of the transverse momentum $\left\langle k_{T}^{2}\left(k_{Z}\right)\right\rangle$ as a function of $k_{z}$ are given below.

$$
\begin{aligned}
P\left(k_{z}\right)_{r f g} d k_{z} & =\frac{3\left(1-k_{z}^{2} / k_{F}^{2}\right)}{4 k_{F}} d k_{z} \\
\left\langle k_{T}^{2}\left(k_{z}\right)_{r f g}\right\rangle & =\frac{1}{2} k_{F}^{2}\left(1-k_{z}^{2} / k_{F}^{2}\right) \\
\left\langle\boldsymbol{k}^{2}\left(k_{z}\right)_{r f g}\right\rangle & =\left\langle k_{T}^{2}\left(k_{z}\right)_{r f g}\right\rangle+k_{z}^{2}
\end{aligned}
$$

\section{B.2 Pauli blocking}

We multiply the QE differetial cross sections by a Pauli blocking factor $K_{\text {Pauli }}^{\text {nuclei }}\left(\boldsymbol{q}_{3}^{2}\right)$ which reduces the predicted cross sections at low $\boldsymbol{q}_{3}^{2}$. The Pauli suppression factor shown below is from Eq. B54 of Ref. [79].

$$
K_{\text {Pauli }}^{\text {nuclei }}=\frac{3}{4} \frac{\left|\boldsymbol{q}_{3}\right|}{k_{F}}\left(1-\frac{1}{12}\left(\frac{\left|\boldsymbol{q}_{3}\right|}{k_{F}}\right)^{2}\right)
$$

For $\left|\boldsymbol{q}_{3}\right|<2 k_{F}$, otherwise no Pauli suppression correction is made. Here $\left|\boldsymbol{q}_{3}\right|=\sqrt{Q^{2}+v^{2}}$ is the absolute magnitude of the 3-momentum transfer to the target nucleus,

\section{Reconstruction of $E_{v}^{Q E-\mu}, Q_{Q E-\mu}^{2}$ and $Q_{Q E-P}^{2}$}

In this section we update the expressions for the mean reconstructed neutrino energy $E_{v}^{Q E-\mu}$ and square of the fourmomentum transfer $Q_{Q E-\mu}^{2}$ extracted only from the kinematics of final state muons in $\mathrm{QE}$ events. In addition we can also reconstruct the four momentum transfer $Q_{Q-(P, N)}^{2}$ from the kinematics of the final state recoil proton or neutron in QE events.

The expressions are updated to include:

1. The contribution of final state interaction $\left|U_{F S I}\right|$.

2. The contribution of Coulomb corrections $V_{\text {eff }}$.
3. The contribution of the proton and neutron transverse momentum $\boldsymbol{k}_{T}$ at the location of the QE peak.

In the derivation of the expressions we use relativistic kinematics. The "primed" energies and momenta are at the vertex before FSI with the nuclear and Coulomb field.

$$
\begin{aligned}
E_{i}^{N, P} & =M_{N, P}-\epsilon^{N, P} \\
E_{f}^{P} & =\sqrt{\left.\left(\boldsymbol{k}+\boldsymbol{q}_{3}\right)^{2}+M_{P}^{2}\right)}-\left|U_{F S I}\right|+\left|V_{e f f}^{P}\right| \\
& =E_{F}^{P^{\prime}}-\left|U_{F S I}\right|+\left|V_{e f f}^{P}\right| \\
E_{f}^{N} & =\sqrt{\left.\left(\boldsymbol{k}+\boldsymbol{q}_{3}\right)^{2}+M_{N}^{2}\right)}-\left|U_{F S I}\right| \\
& =E_{F}^{N \prime}-\left|U_{F S I}\right| \\
E_{\mu-} & =E_{\mu-}^{\prime}-\left|V_{e f f}^{P}\right|, \quad E_{\mu+}=E_{\mu+}^{\prime}+\left|V_{e f f}\right|
\end{aligned}
$$

For neutrino scattering on bound neutrons $\left|V_{e f f}^{P}\right|=\left|V_{e f f}\right|$. We define $M_{N}, M_{P}, m_{\mu}$ as the neutron, proton and muon masses. At the peak location of the $\mathrm{QE}$ distribution the bound neutron momentum is perpendicular to $\boldsymbol{q}$ (i.e. $k_{z}=0$ ). In this case, the average of the square of transverse momenta of the neutron (proton) for a Fermi gas momentum distribution (and also for a Gaussian distribution) is $\left\langle\boldsymbol{k}_{T-N}^{2}\right\rangle=\frac{\left(k_{F}^{N}\right)^{2}}{2^{2}}$ for a bound neutron in the initial state and $\left.\left\langle\boldsymbol{k}_{T-P}^{2}\right\rangle=\frac{\left(k_{F}^{P}\right)^{2}}{2}\right)$ for bound proton in the initial state.

\section{C.1 Using only the kinematics of the $\mu^{-}$}

For neutrino QE events we define $E_{\mu-}^{\prime}=T_{\mu-}+m_{\mu}+\left|V_{e f f}\right|$ as the total Coulomb corrected muon energy. We define $\left(M_{P}^{\prime}\right)^{2}=M_{P}^{2}+\left\langle\boldsymbol{k}_{T-N}^{2}\right\rangle$ to account for the fact that the final state proton has the same average transverse momentum as that of the initial state neutron $\left\langle\boldsymbol{k}_{T-N}^{2}\right\rangle$ with respect to the neutrino-muon scattering plane. From energy-momentum conservation we get:

$$
\begin{aligned}
E_{v}= & p_{\mu-}^{\prime} \cos \theta_{\mu-}+P_{P}^{\prime} \cos \theta_{P} \\
p_{\mu-}^{\prime} \sin \theta_{\mu}= & P_{P}^{\prime} \sin \theta_{P} \\
E_{v}+M_{N}-\epsilon^{N}= & {\left[\sqrt{\left(P_{P}^{\prime}\right)^{2}+\left(M_{P}^{\prime}\right)^{2}}\right]-\left|U_{F S I}\right|+\left|V_{e f f}^{P}\right| } \\
& +E_{\mu-}^{\prime}-\left|V_{e f f}\right| \\
E_{v}+M_{N}^{\prime \prime}= & \sqrt{\left(P_{P}^{\prime}\right)^{2}+\left(M_{P}^{\prime}\right)^{2}}+E_{\mu-}^{\prime} \\
M_{N}^{\prime \prime}= & M_{N}-\left(\epsilon^{N}-\left|U_{F S I}\right|\right) .
\end{aligned}
$$

Here. for neutrino scattering on bound neutrons $\left|V_{e f f}^{P}\right|=$ $\left|V_{e f f}\right|,\left|U_{F S I}=\right| U_{F S I}\left(\boldsymbol{q}_{3}^{2}+\frac{1}{2} k_{F}^{2}\right) \mid, p_{\mu-}^{\prime}=\sqrt{\left(E_{\mu-}^{\prime}\right)^{2}-m_{\mu}^{2}}$, $E_{\mu-}=E_{\mu-}^{\prime}-\left|V_{e f f}\right|, P_{P}^{\prime}$ is the momentum of the final state proton (before FSI) in the neutrino - muon plane, and $\theta_{P}$ is the angle of the proton in the neutrino - muon scattering 
plane. From Eq. 39 we obtain the following expressions.

$$
\begin{aligned}
E_{\nu}^{Q E-\mu} & =\frac{2\left(M_{N}^{\prime \prime}\right) E_{\mu-}-\left(\left(M_{N}^{\prime \prime}\right)^{2}+m_{\mu}^{2}-\left(M_{P}^{\prime}\right)^{2}\right)}{\left.2 \cdot\left[\left(M_{N}^{\prime \prime}\right)-E_{\mu-}+\sqrt{\left(E_{\mu-}\right)^{2}-m_{\mu}^{2}}\right) \cos \theta_{\mu-}\right]} \\
Q_{Q E-\mu}^{2} & =-m_{\mu}^{2}+2 E_{\nu}^{Q E}\left(E_{\mu-}^{\prime}-\sqrt{\left(E_{\mu-}^{\prime}\right)^{2}-m_{\mu}^{2}} \cos \theta_{\mu-}\right) . \\
\boldsymbol{q}_{\mathbf{3}}^{\mathbf{2}} & =Q^{2}+\left(E_{\nu}^{Q E-\mu}-E_{\mu-}\right)^{2}
\end{aligned}
$$

Note that because $\left|U_{F S I}\left(\boldsymbol{q}_{3}^{2}+\frac{1}{2} k_{F}^{2}\right)\right|$ is $\boldsymbol{q}_{3}^{2}$ dependent, the above expressions should be solved iteratively, or an average value corresponding to the mean $\boldsymbol{q}_{3}^{2}$ should be used.

\section{C.2 Using only the kinematics of the $\mu^{+}$}

For antineutrino $\mathrm{QE}$ events we define $E_{\mu+}^{\prime}=T_{\mu+}+m_{\mu}-$ $\left|V_{e f f}\right|$ as the total Coulomb corrected muon energy. We define $\left(M_{N}^{\prime}\right)^{2}=M_{N}^{2}+\left\langle\boldsymbol{k}_{T-P}^{2}\right\rangle$ to account for the fact that the final state neutron has the same average transverse momentum as that of the initial state proton $\left\langle\boldsymbol{k}_{T-P}^{2}\right\rangle$ with respect to the antineutrino - muon scattering plane. From energymomentum conservation we get:

$$
\begin{aligned}
E_{\bar{v}}= & p_{\mu+}^{\prime} \cos \theta_{\mu+}+P_{N}^{\prime} \cos \theta_{N} \\
p_{\mu+}^{\prime} \sin \theta_{\mu}= & P_{N}^{\prime} \sin \theta_{N} \\
E_{\bar{v}}+M_{P}-\epsilon^{P}= & {\left[\sqrt{\left(P_{N}^{\prime}\right)^{2}+\left(M_{N}^{\prime}\right)^{2}}\right]-\left|U_{F S I}\right| } \\
& +E_{\mu+}^{\prime}+\left|V_{e f f}\right| \\
E_{\bar{v}}+M_{P}^{\prime \prime}= & \sqrt{\left(P_{N}^{\prime}\right)^{2}+\left(M_{N}^{\prime}\right)^{2}}+E_{\mu+}^{\prime} \\
M_{P}^{\prime \prime}= & M_{P}-\left(\epsilon^{P}-\left|U_{F S I}\right|+\left|V_{e f f}\right|\right) .
\end{aligned}
$$

Here, $\left|U_{F S I}=\right| U_{F S I}\left(\boldsymbol{q}_{3}^{2}+\frac{1}{2} k_{F}^{2}\right) \mid, p_{\mu+}=\sqrt{\left(E_{\mu+}^{2}-m_{\mu}^{2}\right.}$, $E_{\mu+}=E_{\mu+}^{\prime}+\left|V_{e f f}\right|, P_{N}^{\prime}$ is the momentum of the final state neutron (before FSI) in the antineutrino-muon scattering plane, and $\theta_{N}$ is the angle of the neutron in the neutrino-muon plane. From Eq. 40 we obtain the following expressions.

$$
\begin{aligned}
E_{\bar{v}}^{Q E-\mu+} & =\frac{2\left(M_{P}^{\prime \prime}\right) E_{\mu+}^{\prime}-\left(\left(M_{P}^{\prime \prime}\right)^{2}+m_{\mu}^{2}-\left(M_{N}^{\prime}\right)^{2}\right)}{\left.2 \cdot\left[\left(M_{P}^{\prime \prime}\right)-E_{\mu+}^{\prime}+\sqrt{\left(E_{\mu+}^{\prime}\right)^{2}-m_{\mu}^{2}}\right) \cos \theta_{\mu+}\right]} \\
Q_{Q E-\mu+}^{2} & =-m_{\mu}^{2}+2 E_{\bar{v}}^{Q E}\left(E_{\mu+}^{\prime}-\sqrt{\left(E_{\mu+}^{\prime}\right)^{2}-m_{\mu}^{2}} \cos \theta_{\mu+}\right) . \\
\boldsymbol{q}_{\mathbf{3}}^{\mathbf{2}} & =Q^{2}+\left(E_{\bar{v}}^{Q E-\mu+}-E_{\mu+}\right)^{2}
\end{aligned}
$$

Note that because $\left.\mid U_{F S I}\left(\boldsymbol{q}_{3}^{2}\right)+\frac{1}{2} k_{F}^{2}\right) \mid$ is $\boldsymbol{q}_{3}^{2}$ dependent, the above expressions should be solved iteratively, or an average value corresponding to the mean $\boldsymbol{q}_{3}^{2}$ should be used.

\section{C.2.1 Using only the kinematics of the final state nucleon}

For neutrino $\mathrm{QE}$ events the average reconstructed $Q_{Q E-P}^{2}$ can be extracted from final state proton variables only by using following expression:

$$
\begin{aligned}
M_{P}^{2}= & \left(q+E_{i}^{N}\right)^{2}=-Q^{2}+2\left(M_{N}-\epsilon^{N}\right) v \\
& +\left(M_{N}-\epsilon^{N}\right)^{2} \\
Q_{Q E-P}^{2}= & \left(M_{N}-\epsilon^{N}\right)^{2}-M_{P}^{2} \\
& +2\left(M_{N}-\epsilon^{N}\right)\left[M_{P}+T^{P}-\left(M_{N}-\epsilon^{N}\right)\right]
\end{aligned}
$$

For antineutrino QE events the average reconstructed $Q_{Q E-N}^{2}$ can be extracted from final state neutron variables only by using following expression:

$$
\begin{aligned}
M_{N}^{2}= & \left(q+E_{i}^{P}\right)^{2}=-Q^{2}+2\left(M_{P}-\epsilon^{P}\right) v \\
& +\left(M_{P}-\epsilon^{N}\right)^{2} \\
Q_{Q E-N}^{2}= & \left(M_{P}-\epsilon^{P}\right)^{2}-M_{N}^{2} \\
& +2\left(M_{P}-\epsilon^{P}\right)\left[M_{N}+T^{N}-\left(M_{P}-\epsilon^{P}\right)\right]
\end{aligned}
$$

\section{C.3 Comparison to previous analyses}

If we set $\boldsymbol{k}_{T}^{2}=0, U_{F S I}=0$, and $\left|V_{e f f}\right|=0$, the above equations are reduced to the equations used in previous analyses except that $x^{\nu}$ and $x^{\bar{v}}$ (equation 24) are used.

\section{References}

1. C. Andreopoulos [GENIE], Acta Phys. Polon. B 40, 2461 (2009)

2. C. Andreopoulos, Nucl. Instr. Meth. A 614, 87 (2010)

3. H. Gallagher (NEUGEN), Nucl. Phys. Proc. Suppl. 112 (2002)

4. Y. Hayato (NEUT), Nucl. Phys. Proc. Suppl. 112, 171 (2002)

5. J. Sobczyk (NuWro), PoS nUfaCt 08, 141 (2008)

6. C. Juszczak, Acta Phys. Pol. B 40, 2507 (2009). http://borg.ift.uni. wroc.pl/nuwro/

7. T. Leitner, O. Buss, L. Alvarez-Ruso, U. Mosel (GiBUU), Phys. Rev. C 79, 034601 (2009) arXiv:0812.0587

8. O. Benhar, P. Huber, C. Mariani, D. Meloni, Phys. Rep. 700, 1 (2017)

9. O. Benhar, D. day, I. Sick, Rev. Mod. Phys. 80, 189 (2008)

10. O. Benhar, S. Fantoni, G. Lykasov, Eur. Phys. J. A 7(3), 415 (2000)

11. O. Benhar et al., Phys. Rev. C 55, 244 (1997)

12. A.M. Ankowski, J.T. Sobczyk, Phys. Rev. C 74, 054316 (2006)

13. S.X. Nakamura, Rep. Prog. Phys. 80, 056301 (2017)

14. A.M. Ankowski, O. Benhar, M. Sakuda, Phys. Rev. D 91, 033005 (2015)

15. O. Benhar, Phys. Rev. C 87, 024606 (2013)

16. Y. Horikawa et al., Phys. Rev. C 22, 1680 (1980)

17. E.J. Moniz et al., Phys. Rev. Lett. 26, 445 (1971)

18. E.J. Moniz, Phys. Rev. 184, 1154 (1969)

19. R.R. Whitney, Phys. Rev. C 9, 2230 (1974)

20. R.A. Smith, E.J. Moniz, Nucl. Phys. B 43, 605 (1972)

21. S. Dennis (T2K), Talk at NUFACT, Virginia Tech, Blacksburg, VA (2018) https://indico.phys.vt.edu/event/34/contributions/610/

22. S. Bienstock (2018). Studying the impact of neutrino cross-section mismodelling on the T2K oscillation analysis. https://zenodo.org/ record/1300504

23. S. Bienstock (2018). NEUTRINO 2018 poster https://indico.desy.de/ indico/event/18342/session/35/contribution/173

24. P. Gueye et al., Phys. Rev. C 60, 044308 (1999) 
25. C.A. Ternes, Talk at NUFACT, Virginia Tech, Blacksburg, VA (2018). https://indico.phys.vt.edu/event/34/contributions/760/

26. C. Maieron, T.W. Donnelly, I. Sick, Phys. Rev. C 65, 025502 (2002)

27. J.E. Amaro et al., Phys. Rev. C 71, 015501 (2005)

28. A. Bodek, M.E. Christy, B. Coppersmith, Eur. Phys. J. C 74, 3091 (2014)

29. W.M. Seif, H. Mansour, Int. J. Mod. Phys. E 24, 1550083 (2015)

30. J. Chen, Nuclear Data Sheets 140, 1 (2017); http://www.tunl.duke. edu/nucldata; http://www.nndc.bnl.gov/nudat2/

31. Nuclear Data Tables. www.periodicTable.com/Isotopes/083.208/ index.html; https://www-nds.iaea.org

32. O. Hen, G.A. Miller, E. Piasetzky, L.B. Weinstein, Rev. Mod. Phys. 89(4), 045002 (2017)

33. B.A. Brown, Prog. Part. Nucl. Phys. 47, 517 (2001)

34. B. Frois, I. Sick (Eds.) Modern Topics in Electron Scattering (World Scientific, Singapore, 1991)

35. A. Bodek, J.L. Ritchie, Phys. Rev. D 23, 1070 (1981)

36. D. Koltun, Phys. Rev. Lett. 28, 182 (1972)

37. D. Dutta et al, (Jlab Hall C), Phys. Rev. C 68, 064603 (2003)

38. D. Dutta, PhD Thesis, Northeastern U. (1999)

39. J. Mougey, M. Bernheim, A. Bussire, A. Gillebert, X.H. Phan, M. Priou, D. Royer, I. Sick, G.J. Wagner, Nucl. Phys. A 262, 461 (1976)

40. S. Frullani, J. Mougey, Adv. Nucl. Phys. 14, 1 (1982)

41. K. Nakamura, S. Hiramatsu, T. Kamae, H. Muramatsu, Y. Watase, Nucl. Phys. A 268, 381 (1976)

42. K. Nakamura, S. Hiramatsu, T. Kamae, H. Muramatsu, Y. Watas, Nucl. Phys. A 296, 431 (1978)

43. K. Nakamura, S. Hiramatsu, T. Kamae, H. Muramatsu, Y. Watase, Nucl. Phys. A 271, 221 (1976)

44. P. K. A. de Witt Huberts, J. Phys. G. Nucl. Part. Phys. 507 (1990)

45. A.E.L. Dieperik, P.K.A. de Witt Huberts, Annu. Rev. Nucl. Part Sci. 40, 239 (1990)

46. G. van der Steenhove et al., Nucl. Phys. A 480, 547 (1988)

47. G. van der Steenhove et al., Nucl. Phys. A 484, 445 (1988)

48. N. Liyanage et al, (Jlab Hall A), Phys. Rev. Lett. 86, 5670 (2001)

49. N. Liyanage, PhD Thesis, MIT (1999)
50. K.G. Fissum et al., Phys. Rev. C 70, 034606 (2004)

51. A.M. Ankowski, J.T. Sobczyk, Phys. Rev. C 77, 044311 (2008). arXiv:0711.2031 [nucl-th]

52. Vautherin, D.M. Brink, Phys. Rev. C 5, 626 (1972)

53. Quasielastic Electron Nucleus Scattering Archive: http://faculty. virginia.edu/qes-archive/

54. O. Benhar, D. Day, I. Sick, Rev. Mod. Phys. Rev. Mod. Phys. 80, 189-224 (2008)

55. F.H. Heimlich, Nucl. Phys. A 231, 509 (1979)

56. F.H. Heimlich et al., Nucl. Phys. A 231, 509 (1974)

57. R.R. Whitney, Phys. Rev. C 9, 2230 (1974)

58. P. Barreau, Nucl. Phys. A 402, 515 (1983)

59. R. Sealock et al., Phys. Rev. Lett. 62, 1350-1353 (1989)

60. D. Baran et al., Phys. Rev. Lett. 61, 400-403 (1988)

61. D.S. Bagdasaryan, et al, YERPHI-1077-40-88

62. D. Zeller, DESY Internal Report F23-73/2 (1973)

63. F.H. Heimlich et al. DESY Report 74/20 (1974)

64. J. Arrington, Phys. Rev. C 53, 2248 (1996)

65. N. Fomin et al., Phys. Rev. Lett. 105, 212502 (2010)

66. M. Anghinolfi et al., Nucl. Phys. A 602, 405 (1996)

67. J.S. O'Connell, Phys. Rev. C 35, 1243 (1987)

68. H. Dai et al arXiv:1810.10575 [nucl-ex]

69. M. Anghinolfi et al., J. Phys. G Nucl. Pan. Phys. 21, L9-L15 (1995)

70. P.Y. Bosted et al., Phys. Rev. C 46, 2505 (1992). (Steve Rock, Private Commun. (A127 Rock-pc))

71. C.F. Williamson et al., Phys. Rev. C M 56, 3152 (1997)

72. T.C. Yates et al., Phys. Lett. B 312, 382 (1993)

73. A. Hotta et al., Phys. Rev. C 30, 87 (1984)

74. Z.E. Meziani et al., Phys. Rev. Lett. 52, 2130 (1984)

75. J. Arrington et al., Phys. Rev. Lett. 82, 20562059 (1999)

76. J.P. Chen, Phys. Rev. Lett. 66, 1283 (1991)

77. D.B. Day, Phys. Rev. C 48, 1849 (1993)

78. A. Zghiche et al., Nucl. Phys. A 572, 513 (1994)

79. Y.S. Tsai, Rev. Mod. Phys. 46(815), 123 (1974) 